\title{
Analysis of Leptosphaeria maculans Race Structure in a Worldwide Collection of Isolates
}

\author{
M. H. Balesdent, M. J. Barbetti, Hua Li, K. Sivasithamparam, L. Gout, and T. Rouxel
}

First, fifth, and sixth authors: Pathologie Végétale et Méthodologies de la Détection (PMDV), INRA, Route de Saint Cyr, 78026 Versailles Cedex, France; second author: School of Plant Biology, and third and fourth authors: School of Earth and Geographical Sciences, Faculty of Natural and Agricultural Sciences, The University of Western Australia, Crawley, W.A. 6009, Australia.

Accepted for publication 11 May 2005.

\begin{abstract}
Balesdent, M. H., Barbetti, M. J., Li, H., Sivasithamparam, K., Gout, L., and Rouxel, T. 2005. Analysis of Leptosphaeria maculans race structure in a worldwide collection of isolates. Phytopathology 95:1061-1071.

Leptosphaeria maculans, the causal agent of stem canker of oilseed rape, develops gene-for-gene interactions with its hosts. To date, eight $L$. maculans avirulence (Avr) genes, AvrLm1 to AvrLm8, have been genetically characterized. An additional Avr gene, AvrLm9, that interacts with the resistance gene $R \operatorname{lm} 9$, was genetically characterized here following in vitro crosses of the pathogen. A worldwide collection of 63 isolates, including the International Blackleg of Crucifers Network collection, was

of each PG. Genotyping of isolates at all Avr loci confirmed the disparities between continents, in terms of Avr allele frequencies, particularly for AvrLm2, AvrLm3, AvrLm7, AvrLm8, and AvrLm9, or in terms of race structure, diversity, and complexity. Twenty-six distinct races were identified in the collection. A larger number of races $(n=18)$ was found in Australia than in Europe $(n=8)$. Mean number of virulence alleles per isolate was also higher in Australia (5.11 virulence alleles) than in Europe (4.33) and Canada (3.46). Due to the diversity of populations of $L$. maculans evidenced here at the race level, a new, open terminology is proposed for L. maculans race designation, indicating all Avr loci for which the isolate is avirulent.
\end{abstract} genotyped at these nine Avr loci. In a first step, isolates were classified into pathogenicity groups (PGs) using two published differential sets. This analysis revealed geographical disparities as regards the proportion
Additional keywords: Brassica napus, IBCN, specific resistance.
The field resistance of oilseed rape, Brassica napus, to the fungal pathogen Leptosphaeria maculans, causing stem canker of crucifers, is known to combine both quantitative, polygenic resistance and specific, major-gene, resistance (13). In the last decade, efforts were developed to determine the genetic bases of the genefor-gene interactions expressed at the leaf level between $L$. maculans and its host plants, B. napus, B. rapa, and B. juncea $(5,8$, $9,13,15,35)$. A set of three host differentials (B. napus cvs. Westar, Quinta, and Glacier) originally was used to classify isolates of L. maculans into one of three pathogenicity groups (PGs) (PG2 to PG4) $(23,32)$, that each could be further subdivided into two groups using the additional differential cv. Jet Neuf $(7,24)$. The usefulness of this improved differential set to classify isolates of L. maculans into one of the six corresponding races, termed A1 to A6 (24), was supported by genetic evidence that each of these races corresponded to different combinations of alleles of three avirulence (Avr) genes of the pathogen, namely AvrLm1, AvrLm2, and AvrLm4 (4,5,8,42). More recently, further genetic studies involving additional specific interactions between $L$. maculans and $B$. napus, B. rapa, or B. juncea, demonstrated that at least six distinct $A v r$ genes governed the recognition between $L$. maculans and its hosts (9). Consequently, up to $64 \mathrm{~L}$. maculans races theoretically could be observed in the field, which renders obsolete the first classifications into three to six races. Genetic evidence of the occurrence of dominant, single resistance $(R)$ genes in the host, matching Avr alleles, also was reported $(5,8,13$, $15,21,28)$.

Corresponding author : M. H. Balesdent; E-mail address: mhb@versailles.inra.fr

DOI: 10.1094/PHYTO-95-1061

(C) 2005 The American Phytopathological Society
In parallel to these genetic studies, it was demonstrated that $R$ genes exerted a strong selection pressure on populations of L. maculans. This was the case either following the large-scale use of one $R$ gene in agronomic field conditions in France (AvrLm1-Rlm1 interaction) or following the recurrent use of one $R$ gene in experimental fields (AvrLm6-Rlm6 interaction) $(12,40)$. This selection pressure induced a very rapid shift of the pathogen population structure from avirulence to virulence, with the corresponding loss of efficiency of the $R$ gene in the agricultural practice (40). Similarly, in Australia, L. maculans populations recently overcame the single dominant $B$. rapa subsp. sylvestris derived resistance $(19,20,29)$, a resistance utilized widely in Australia (3). Hence, both genetic studies performed under controlled conditions at the earliest stage of infection, using the miniaturized cotyledon inoculation test, and field observations of the shift from avirulent to virulent populations in experimental or natural field conditions demonstrated that, as already described in most pathosystems, gene-for-gene host-pathogen interactions involving $R$ and Avr genes are the rule in the L. maculans-Brassica sp. system.

In a perspective of a durable management of the few specific resistance sources described in Brassica spp. toward L. maculans, as well as to take into account the potential risk of introducing new virulent races in specific locations where they may be absent, it is now a priority to re-examine our knowledge of race structure of L. maculans. As a first step toward this goal, worldwide isolates were characterized for their Avr alleles composition, taking into account all currently described $A v r / R$ gene pairs. This analysis included historical isolates of the International Blackleg of Crucifers Network (IBCN) collection, composed of isolates from the main regions where this pathogen is a threat, i.e., Canada, Europe, and Australia (41). This article reports on the validation of the plant differentials to characterize isolates of L. maculans in 
terms of Avr alleles, and describes disparities between continents as regards Avr allele frequencies and race structure. In the course of this characterization, the putative additional Avr gene AvrLm9 (13) was identified genetically and new putative specific interactions were evidenced. Finally, we propose a renewed terminology for races of L. maculans that precisely describes the Avr allele composition of each race, and that may evolve as soon as new Avr genes are described, because more $A v r / R$ gene pairs are likely to occur in this pathosystem.

\section{MATERIALS AND METHODS}

Fungal strains and culture maintenance. The IBCN collection comprises 93 isolates $(31,37,41,46)$. From these, 55 isolates belong to the $L$. maculans sensu stricto (the A/Tox ${ }^{+}$) species, termed L. maculans "brassicae" (31) and hereafter termed L. maculans. Twelve of these isolates, that sporulated poorly, could not be inoculated consistently and, thus, were excluded from this study. The 43 remaining IBCN isolates analyzed originated from Europe (19 isolates), Canada (13 isolates), and Australia (11 isolates) (Table 1). This collection was completed by isolates Nz-T4 (9) and Nz-G (31), originating from New Zealand, 12 isolates collected in Australia from 1971 to 2002 (Table 1) $(19,20)$, and the PG4 reference isolates PHW1223 and H5 (4,35). Isolate BBA62908 is one of the oldest European isolates available (42) (Table 1). To increase the geographic diversity of European iso- lates, three European isolates, UK1, PT1, and Raw4, also were used $(6,22,46)$. All these field isolates originated from $B$. napus, except isolates IBCN51-59 and UWAM3, isolated from B. juncea, IBCN74 isolated from B. oleracea (31), and WAC7803, isolated from Raphanus raphanistrum growing alongside of blackleginfested oilseed rapeseed crops. Fungal culture conditions for sporulation were as reported previously (4). For long-term conservation, isolates were stored at $4^{\circ} \mathrm{C}$, either in $1 \%$ malt-agar slant tubes or as small pieces of V8 agar, cut from actively growing cultures, and dipped in sterile mineral oil.

In vitro crossing. In order to genetically identify the $A v r$ gene inducing resistance on cultivars possessing the $R$ gene $\operatorname{Rlm} 9$ (13), two fungal crosses were performed: cross 39 (IBCN59 $\times$ H5) and cross 58 (PHW1223 $\times$ v23.1.2). In vitro crosses and random ascospore progeny recovery were performed as previously established (18).

Plant material. Isolates were inoculated on (i) cultivars or lines belonging to the published differential set 1 , comprising Westar, QuintaV (Rlm1, Rlm4), Glacier $(R \operatorname{lm} 2, R \operatorname{lm} 3)$, and Jet Neuf $(R \operatorname{lm} 4)(7,23,24,32)$; (ii) the differential set 2 comprising Westar, Bristol (Rlm2), Columbus (Rlm1, Rlm3), and Jet Neuf $(9,40,42)$; (iii) the additional differential lines described by Balesdent et al. (9), possessing $R$ genes $R \operatorname{lm} 3$ (line 22-1-1), Rlm5 (lines 150-2-1 and 151-2-1), Rlm6 (line Falcon-MX), Rlm5 + Rlm6 (B. juncea cv. Picra), Rlm7 (line 23-1-1), or $R \operatorname{lm} 8$ (line 156-2-1); (iv) additional cultivars used for an independent genotyping of Avr

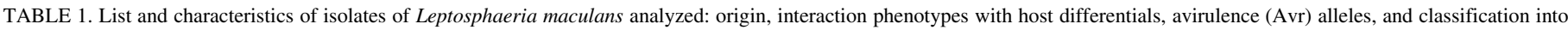
pathogenicity groups (PGs) and races

\begin{tabular}{|c|c|c|c|c|c|c|c|c|c|c|c|c|c|c|c|c|c|c|c|c|c|c|c|c|c|c|c|c|c|c|c|}
\hline \multirow[b]{3}{*}{ Isolate } & \multirow[b]{3}{*}{ Other name } & \multirow[b]{3}{*}{ Year $^{\mathrm{a}}$} & \multirow[b]{3}{*}{ Origin $^{b}$} & \multirow[b]{3}{*}{ Q } & \multirow{2}{*}{\multicolumn{13}{|c|}{ Interaction phenotypes on the plant differential line ${ }^{c}$}} & \multicolumn{4}{|c|}{ Classification $^{\mathrm{d}}$} & & & & & & & & & & \multirow[b]{3}{*}{ Race $^{f}$} \\
\hline & & & & & & & & & & & & & & & & & & \multicolumn{2}{|c|}{ Set 1} & \multicolumn{2}{|c|}{ Set 2} & \multicolumn{9}{|c|}{ Genotype AvrLm 1 to $9^{\mathrm{e}}$} & \\
\hline & & & & & Gl & $\mathrm{J}$ & $\mathrm{C}$ & $\mathrm{B}$ & $S$ & Go & $\mathrm{Da}$ & $\mathrm{P}$ & 22 & 23 & 150 & 156 & $\mathrm{DM}$ & PG & A & PG & A & 1 & 2 & 3 & 4 & 5 & 6 & 7 & 8 & 9 & \\
\hline BBA62908 & & 1966 & $\mathrm{E}(\mathrm{G})$ & A & $\mathrm{A}$ & $\mathrm{A}$ & A & A & A & $\mathrm{V}$ & $\mathrm{V}$ & A & $\mathrm{V}$ & $\mathrm{A}$ & A & A & A & 2 & 3 & 2 & 3 & + & + & - & + & + & + & + & + & - & Av1-2-4-5-6-7-8 \\
\hline IBCN01 & IIA1 & 1996 & $E(G)$ & $\mathrm{V}$ & $\mathrm{V}$ & $\mathrm{V}$ & $\mathrm{V}$ & $\mathrm{V}$ & $\mathrm{V}$ & $\mathrm{V}$ & $\mathrm{V}$ & $\mathrm{V}$ & $\mathrm{V}$ & $\mathrm{A}$ & A & A & A & 4 & 1 & 4 & 1 & - & - & - & - & + & + & + & + & - & Av5-6-7-8 \\
\hline IBCNO2 & $\mathrm{T} 12 \mathrm{aD} 34$ & 1992 & $E(G)$ & $\mathrm{V}$ & $\mathrm{V}$ & $\mathrm{V}$ & $\mathrm{V}$ & $\mathrm{V}$ & $\mathrm{V}$ & $\mathrm{V}$ & $\mathrm{V}$ & $\mathrm{V}$ & $\mathrm{V}$ & $\mathrm{A}$ & A & A & A & 4 & 1 & 4 & 1 & - & - & - & - & + & + & + & + & - & Av5-6-7-8 \\
\hline IBCN03 & $\mathrm{T} 11 \mathrm{aB} 4$ & 1991 & $\mathrm{E}(\mathrm{G})$ & A & $\mathrm{V}$ & $\mathrm{V}$ & $\mathrm{A}$ & $\mathrm{V}$ & $\mathrm{V}$ & $\mathrm{V}$ & $\mathrm{V}$ & $\mathrm{V}$ & $\mathrm{V}$ & A & A & $\mathrm{V}$ & A & 3 & 2 & 3 & 2 & + & - & - & - & + & + & + & - & - & Av1-5-6-7 \\
\hline IBCN04 & $\mathrm{T} 11 \mathrm{aD} 18$ & 1992 & $E(G)$ & A & $\mathrm{V}$ & $\mathrm{V}$ & $\mathrm{A}$ & $\mathrm{V}$ & $\mathrm{V}$ & $\mathrm{V}$ & $\mathrm{V}$ & $\mathrm{V}$ & $\mathrm{V}$ & $\mathrm{A}$ & A & A & A & 3 & 2 & 3 & 2 & + & - & - & - & + & + & + & + & - & Av1-5-6-7-8 \\
\hline IBCN05 & $\mathrm{T} 12 \mathrm{aC} 25$ & 1992 & $E(G)$ & A & $\mathrm{V}$ & A & $\mathrm{V}$ & $\mathrm{V}$ & $\mathrm{V}$ & $\mathrm{V}$ & $\mathrm{V}$ & A & $\mathrm{V}$ & $\mathrm{A}$ & A & A & A & 3 & 6 & 4 & 5 & - & - & - & + & + & + & + & + & - & Av4-5-6-7-8 \\
\hline IBCN07 & T19bG31 & 1993 & $E(G)$ & $\mathrm{V}$ & $\mathrm{V}$ & $\mathrm{V}$ & $\mathrm{V}$ & $\mathrm{V}$ & $\mathrm{V}$ & $\mathrm{V}$ & $\mathrm{V}$ & $\mathrm{V}$ & $\mathrm{V}$ & $\mathrm{A}$ & A & A & A & 4 & 1 & 4 & 1 & - & - & - & - & + & + & + & + & - & Av5-6-7-8 \\
\hline IBCN13 & 1.1 & 1991 & $\mathrm{~A}(\mathrm{~V})$ & $\mathrm{V}$ & $\mathrm{A}$ & V & V & A & A & A & $\mathrm{A}$ & $\mathrm{V}$ & $\mathrm{V}$ & $\mathrm{V}$ & $\mathrm{A}$ & $\mathrm{V}$ & ND & $X$ & $\mathrm{X}$ & $\mathrm{X}$ & $X$ & - & + & - & - & + & + & - & - & + & Av2-5-6-9 \\
\hline IBCN14 & MD2 & 1988 & $\mathrm{~A}(\mathrm{~S})$ & $\mathrm{V}$ & $\mathrm{V}$ & $\mathrm{V}$ & $\mathrm{V}$ & $\mathrm{V}$ & $\mathrm{V}$ & $\mathrm{V}$ & $\mathrm{V}$ & $\mathrm{V}$ & $\mathrm{V}$ & $\mathrm{V}$ & A & $\mathrm{V}$ & A & 4 & 1 & 4 & 1 & - & - & - & - & + & + & - & - & - & Av5-6 \\
\hline IBCN15 & P9 & 1988 & $\mathrm{~A}(\mathrm{~V})$ & $\mathrm{V}$ & V & $\mathrm{V}$ & V & V & $\mathrm{V}$ & $\mathrm{V}$ & $\mathrm{V}$ & $\mathrm{V}$ & $\mathrm{V}$ & $\mathrm{V}$ & $\mathrm{A}$ & A & A & 4 & 1 & 4 & 1 & - & - & - & - & + & + & - & + & - & Av5-6-8 \\
\hline IBCN16 & 10 & 1988 & $\mathrm{~A}(\mathrm{~W})$ & $\mathrm{V}$ & $\mathrm{V}$ & $\mathrm{V}$ & V & V & $\mathrm{V}$ & $\mathrm{V}$ & $\mathrm{V}$ & $\mathrm{V}$ & $\mathrm{V}$ & $\mathrm{V}$ & A & $\mathrm{V}$ & V & 4 & 1 & 4 & 1 & - & - & - & - & + & - & - & - & - & Av5 \\
\hline IBCN17 & $\mathrm{C} 13$ & 1988 & $\mathrm{~A}(\mathrm{~S})$ & $\mathrm{A}$ & $\mathrm{V}$ & A & $\mathrm{V}$ & V & $\mathrm{V}$ & V & V & A & $\mathrm{V}$ & $\mathrm{A}$ & A & A & A & 3 & 6 & 4 & 5 & - & - & - & + & + & + & + & + & - & Av4-5-6-7-8 \\
\hline IBCN18 & M1 & 1988 & $\mathrm{~A}(\mathrm{~V})$ & A & A & A & A & $\mathrm{A}$ & A & V & $\mathrm{V}$ & A & $\mathrm{V}$ & $\mathrm{A}$ & $\mathrm{V}$ & $\mathrm{V}$ & $\mathrm{V}$ & 2 & 3 & 2 & 3 & + & + & - & + & - & - & + & - & - & Av1-2-4-7 \\
\hline IBCN42 & $\mathrm{C} 2$ & 1990 & $E(F)$ & A & $\mathrm{V}$ & $\mathrm{V}$ & A & $\mathrm{V}$ & $\mathrm{V}$ & $\mathrm{V}$ & $\mathrm{V}$ & $\mathrm{V}$ & $\mathrm{V}$ & $\mathrm{A}$ & A & A & A & 3 & 2 & 3 & 2 & + & - & - & - & + & + & + & + & - & Av1-5-6-7-8 \\
\hline IBCN44 & $\mathrm{F} 1$ & 1990 & $\mathrm{E}(\mathrm{F})$ & A & $\mathrm{V}$ & $\mathrm{V}$ & $\mathrm{A}$ & $\mathrm{V}$ & $\mathrm{V}$ & $\mathrm{V}$ & $\mathrm{V}$ & $\mathrm{V}$ & $\mathrm{V}$ & A & A & A & A & 3 & 2 & 3 & 2 & + & - & - & - & + & + & + & + & - & Av1-5-6-7-8 \\
\hline IBCN45 & $\mathrm{F} 2$ & 1990 & $\mathrm{E}(\mathrm{F})$ & A & $\mathrm{V}$ & $\mathrm{V}$ & A & $\mathrm{V}$ & $\mathrm{V}$ & $\mathrm{V}$ & $\mathrm{V}$ & $\mathrm{V}$ & $\mathrm{V}$ & $\mathrm{A}$ & A & A & A & 3 & 2 & 3 & 2 & + & - & - & - & + & + & + & + & - & Av1-5-6-7-8 \\
\hline IBCN47 & $\ldots$ & 1990 & $E(F)$ & $\mathrm{V}$ & $\mathrm{V}$ & $\mathrm{V}$ & $\mathrm{V}$ & V & $\mathrm{V}$ & $\mathrm{V}$ & $\mathrm{V}$ & $\mathrm{V}$ & $\mathrm{V}$ & A & A & A & A & 4 & 1 & 4 & 1 & - & - & - & - & + & + & + & + & - & Av5-6-7-8 \\
\hline IBCN50 & $\ldots$ & 1990 & $\mathrm{E}(\mathrm{F})$ & A & $\mathrm{V}$ & $\mathrm{V}$ & $\mathrm{A}$ & $\mathrm{V}$ & $\mathrm{V}$ & $\mathrm{V}$ & $\mathrm{V}$ & $\mathrm{V}$ & $\mathrm{V}$ & $\mathrm{A}$ & A & A & A & 3 & 2 & 3 & 2 & + & - & - & - & + & + & + & + & - & Av1-5-6-7-8 \\
\hline IBCN51 & BJ-55 & 1992 & $\mathrm{C}(\mathrm{S})$ & A & $\mathrm{A}$ & A & A & A & A & A & $\mathrm{A}$ & $\mathrm{V}$ & A & $\mathrm{V}$ & A & $\mathrm{V}$ & ND & 2 & 3 & 2 & 3 & + & + & + & - & + & + & - & - & + & Av1-2-3-5-6-9 \\
\hline IBCN52 & BJ-60 & 1992 & $\mathrm{C}(\mathrm{S})$ & A & $\mathrm{A}$ & A & A & A & A & A & $\mathrm{A}$ & $\mathrm{V}$ & A & $\mathrm{V}$ & A & $\mathrm{V}$ & ND & 2 & 3 & 2 & 3 & + & + & + & - & + & + & - & - & + & Av1-2-3-5-6-9 \\
\hline IBCN53 & BJ-109 & 1992 & $\mathrm{C}(\mathrm{S})$ & A & $\mathrm{A}$ & A & A & A & A & A & $\mathrm{A}$ & A & A & $\mathrm{V}$ & A & $\mathrm{V}$ & ND & 2 & 3 & 2 & 3 & + & + & + & + & + & ND & - & - & + & Av1-2-3-4-5-9-(6) \\
\hline IBCN54 & BJ-110 & 1992 & $\mathrm{C}(\mathrm{S})$ & A & $\mathrm{A}$ & A & A & A & A & A & $\mathrm{A}$ & A & A & $\mathrm{V}$ & A & $\mathrm{V}$ & ND & 2 & 3 & 2 & 3 & + & + & + & + & + & ND & - & - & + & Av1-2-3-4-5-9-(6) \\
\hline IBCN55 & BL-113-2 & 1992 & $\mathrm{C}(\mathrm{S})$ & $\mathrm{V}$ & $\mathrm{A}$ & A & A & A & $\mathrm{A}$ & A & $\mathrm{A}$ & $\mathrm{V}$ & A & $\mathrm{V}$ & A & $\mathrm{V}$ & ND & $\mathrm{X}$ & $\mathrm{X}$ & 2 & 3 & - & + & + & - & + & + & - & - & + & Av2-3-5-6-9 \\
\hline IBCN56 & BJ-121 & 1992 & $\mathrm{C}(\mathrm{S})$ & A & A & A & $\mathrm{A}$ & $\mathrm{A}$ & A & A & A & $\mathrm{V}$ & A & $\mathrm{V}$ & A & $\mathrm{V}$ & ND & 2 & 3 & 2 & 3 & + & + & + & - & + & + & - & - & + & $\begin{array}{l}\text { Av1-2-3-5-6-9 } \\
\text { tinued on next pag }\end{array}$ \\
\hline
\end{tabular}

a Year of isolation; ND = not determined

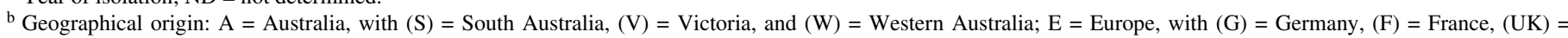
United Kingdom, $(\mathrm{Pt})=$ Portugal, and $(\mathrm{P})=$ Poland; $\mathrm{C}=$ Canada, with $(\mathrm{O})=$ Ontario and $(\mathrm{S})=$ Saskatchewan; NZ $=\mathrm{New} Z$ Zealand; ivc $=$ isolate issued from in vitro cross.

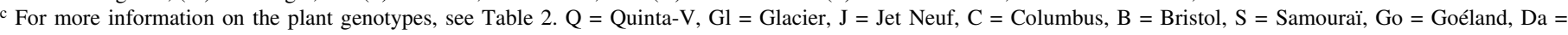
Darmor, $\mathrm{P}=$ Pollen, $22=22-1-1,23=23-2-1,150=150-2-1,156=156-2-1$, DM $=$ Darmor-MX, $\mathrm{A}=$ the isolate is avirulent on the plant genotype, $\mathrm{V}=$ the isolate is virulent, and $\mathrm{ND}=$ not determined.

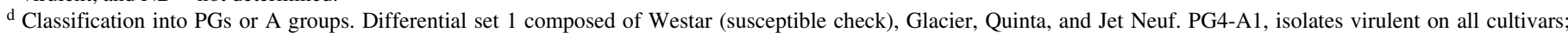
PG4-A5, virulent on all cultivars but Jet Neuf; PG3-A2, virulent on all cultivars but Quinta, PG3-A6, avirulent on Quinta and Jet Neuf, PG2-A4, avirulent on Glacier and Quinta, PG2-A3, avirulent on all cultivars but Westar, X, undescribed group (7,23,32). Differential set 2 composed of Westar (susceptible check), Bristol, Columbus, and Jet neuf. PG4-A1, isolates virulent on all cultivars; PG4-A5, virulent on all cultivars but Jet Neuf; PG3-A2, virulent on all cultivars but Columbus, PG3-A6, avirulent on Columbus and jet Neuf, PG2-A4, avirulent on Bristol and Columbus, PG2-A3, avirulent on all cultivars but Westar, X, undescribed group (9,40,42).

e Genotype of the isolates at each avirulent (Avr) locus; $+=$ presence of the Avr allele, $-=$ presence of the virulent allele, and ND $=$ not determined.

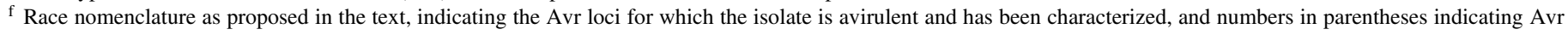
loci for which the allele has not been identified. 
loci and chosen because they possessed $R$ genes identical to those present in the differentials, such as Samouraï (Rlm2), Navajo (Rlm3), Falcon and Pollen (Rlm4), additional Rlm6 lines Samouraï-MX and Darmor-MX, and additional Rlm7 lines 04-12, 148-1-1, and 149-2-1 (9,13,42; T. Rouxel and M. H. Balesdent, unpublished data); and (v) three winter B. napus cultivars putatively possessing the $R$ gene Rlm 9 (i.e., Darmor [13], Goéland, and Mohican [this study]) (Table 2). Seventeen additional wintertype cultivars (i.e., Adélie, Andol, Cando, Capitol, Cheyenne, Coronet, Doublol, Elite, Eurol, Express, Geronimo, Idol, Kintol, Maxol, Symbol, Synergy, and Vivol) also were screened for the presence of Rlm9. Lines or cultivars were obtained as described previously $(8,9,42)$. Seeds of B. napus lines Samouraï-MX and Darmor-MX, harboring the B. juncea gene Rlm6, were provided by M. Renard (INRA, Le Rheu, France).

Pathogenicity tests. Isolates were inoculated on cotyledons of the Brassica genotypes as described previously (13, 17). Spore suspensions $(10 \mu \mathrm{l})$ adjusted at $10^{7}$ spores $\mathrm{ml}^{-1}$ were deposited on each half-cotyledon of 14-day-old plantlets previously wounded with a needle. Plants were incubated in a growth chamber at 16 and $24^{\circ} \mathrm{C}$ (night and day, respectively) with a 12 -h photoperiod. Symptoms were scored two to three times, between 14 and 27 days after inoculation, using a rating scale comprising six infection classes (IC), where IC1 is the typical hypersensitive response, IC2 represents a larger (1.5- to 3-mm) dark necrotic lesion, IC3 is a nonsporulating lesion that is sharply delimited by a dark necrotic margin that may extend within the lesion. IC4 to IC6 are characterized by gray-green tissue collapse without a darkened margin, and showing no sporulation (IC4), a few pycnidia (IC5), or profuse sporulation (IC6) (8). As previously discussed, IC1 to 3 are resistance responses and IC4 to 6 are susceptibility symptoms (8). Each isolate was inoculated on at least 10 different plants, and experiments were repeated at least twice. The result of each isolateline interaction was recorded as compatible or incompatible as previously established $(8,9)$. Each Avr allele was characterized on two distinct plant genotypes (such as Bristol and Samouraï to identify $A v r L m 2$ ), expect in the case of $\operatorname{Rlm} 8$, for which only one plant genotype was available. The reference isolates v11.1.1, v11.1.2, v23.1.2, v23.1.3, v23.2.1, and 22.2.2, for which genotypes at Avr loci AvrLm1 to AvrLm8 were identified previously by genetic analyses, were used as controls in pathogenicity tests $(9,13)$ (Table 1).

Data analyses. The ratio of avirulent:virulent $(\mathrm{A}: \mathrm{V})$ progeny isolates of a given cross was tested for deviation from Mendelian expectation using a $\chi^{2}$ test. For random progeny in which more than two Avr genes segregated, linkage analyses among Avr loci was performed using the Mapmaker/EXP 3.0b software with a log of the likelihood ratios score of 4.0 and a maximum recombination frequency of 30 centimorgans (cM).

Whenever the identification of the allele present at one Avr locus was possible, the data was recorded as 0 (virulent allele) or 1 (avirulent allele). The data matrix obtained for all field isolates

\begin{tabular}{|c|c|c|c|c|c|c|c|c|c|c|c|c|c|c|c|c|c|c|c|c|c|c|c|c|c|c|c|c|c|c|c|}
\hline \multirow[b]{3}{*}{ Isolate } & \multirow[b]{3}{*}{ Other name } & \multirow[b]{3}{*}{ Year $^{\mathrm{a}}$} & \multirow[b]{3}{*}{ Origin $^{b}$} & & & & & & & & & & & & & & & \multicolumn{4}{|c|}{ Classification $^{\mathrm{d}}$} & \multirow{2}{*}{\multicolumn{9}{|c|}{ Genotype AvrLml to $9^{\mathrm{e}}$}} & \multirow[b]{3}{*}{ Race $^{f}$} \\
\hline & & & & \multicolumn{14}{|c|}{ Interaction phenotypes on the plant differential line ${ }^{c}$} & \multicolumn{2}{|c|}{ Set 1} & \multicolumn{2}{|c|}{ Set 2} & & & & & & & & & & \\
\hline & & & & Q & Gl & $\mathrm{J}$ & $\mathrm{C}$ & $\mathrm{B}$ & $\mathrm{S}$ & Go & $\mathrm{Da}$ & $\mathrm{P}$ & 22 & 23 & 150 & 156 & $\mathrm{DM}$ & PG & A & PG & A & 1 & 2 & 3 & 4 & 5 & 6 & 7 & 8 & 9 & \\
\hline IBCN58 & RBJ-56A & 1992 & $\mathrm{C}(\mathrm{S})$ & A & A & A & A & A & $\mathrm{A}$ & $\mathrm{A}$ & A & $\mathrm{V}$ & $\mathrm{A}$ & $\mathrm{V}$ & A & V & ND & 2 & 3 & 2 & 3 & + & + & + & - & + & + & - & - & + & Av1-2-3-5-6-9 \\
\hline IBCN59 & RBJ-135B & 1992 & $\mathrm{C}(\mathrm{S})$ & A & A & A & A & A & A & A & A & V & A & V & A & $\mathrm{V}$ & ND & 2 & 3 & 2 & 3 & + & + & + & - & + & + & - & - & + & Av1-2-3-5-6-9 \\
\hline IBCN66 & $\mathrm{BnA1}$ & 1985 & $E(F)$ & $\mathrm{V}$ & $\mathrm{V}$ & $\mathrm{V}$ & $\mathrm{V}$ & V & $\mathrm{V}$ & $\mathrm{V}$ & V & V & $\mathrm{V}$ & A & A & A & A & 4 & 1 & 4 & 1 & - & - & - & - & + & + & + & + & - & Av5-6-7-8 \\
\hline IBCN68 & $\mathrm{BnA} 3$ & 1990 & $\mathrm{E}(\mathrm{F})$ & A & $\mathrm{V}$ & $\mathrm{V}$ & A & $\mathrm{V}$ & $\mathrm{A}$ & $\mathrm{V}$ & $\mathrm{V}$ & $\mathrm{V}$ & $\mathrm{V}$ & A & A & A & A & 3 & 2 & 3 & 2 & + & - & - & - & + & + & + & + & - & Av1-5-6-7-8 \\
\hline IBCN69 & $\mathrm{BnA} 4$ & 1990 & $E(F)$ & A & $\mathrm{V}$ & V & A & V & $\mathrm{V}$ & $\mathrm{V}$ & V & $\mathrm{V}$ & V & A & A & A & A & 3 & 2 & 3 & 2 & + & - & - & - & + & + & + & + & - & Av1-5-6-7-8 \\
\hline IBCN70 & $\mathrm{BnA5}$ & 1990 & $E(F)$ & A & $\mathrm{V}$ & V & A & V & $\mathrm{V}$ & $\mathrm{V}$ & V & $\mathrm{V}$ & $\mathrm{V}$ & A & $\mathrm{V}$ & A & A & 3 & 2 & 3 & 2 & + & - & - & - & - & + & + & + & - & Av1-6-7-8 \\
\hline IBCN71 & BnA6 & 1990 & $E(F)$ & A & $\mathrm{V}$ & A & A & $\mathrm{V}$ & $\mathrm{V}$ & $\mathrm{V}$ & $\mathrm{V}$ & A & $\mathrm{V}$ & A & A & A & A & 3 & 6 & 3 & 6 & + & - & - & + & + & + & + & + & - & Av1-4-5-6-7-8 \\
\hline IBCN72 & $\mathrm{BnA} 7$ & 1990 & $\mathrm{E}(\mathrm{F})$ & A & $\mathrm{V}$ & V & A & V & $\mathrm{V}$ & V & V & V & V & A & A & A & A & 3 & 2 & 3 & 2 & + & - & - & - & + & + & + & + & - & Av1-5-6-7-8 \\
\hline IBCN73 & PHW1243 & ND & $E(G)$ & A & $\mathrm{V}$ & $\mathrm{V}$ & A & V & $\mathrm{V}$ & $\mathrm{V}$ & V & $\mathrm{V}$ & $\mathrm{V}$ & A & A & $\mathrm{V}$ & A & 3 & 2 & 3 & 2 & + & - & - & - & + & + & + & - & - & Av1-5-6-7 \\
\hline IBCN74 & PHW1245 & ND & $E(F)$ & A & A & A & A & A & A & V & $\mathrm{V}$ & A & V & A & A & A & A & 2 & 3 & 2 & 3 & + & + & - & + & + & + & + & + & - & Av1-2-4-5-6-7-8 \\
\hline IBCN75 & PHW1276 & 1987 & $\mathrm{~A}(\mathrm{~W})$ & A & $\mathrm{V}$ & $\mathrm{V}$ & A & V & $\mathrm{V}$ & $\mathrm{V}$ & V & V & $\mathrm{V}$ & V & A & A & A & 3 & 2 & 3 & 2 & + & - & - & - & + & + & - & + & - & Av1-5-6-8 \\
\hline IBCN76 & PHW1278 & 1987 & $\mathrm{~A}(\mathrm{~W})$ & A & A & $\mathrm{V}$ & A & $\mathrm{V}$ & $\mathrm{V}$ & $\mathrm{V}$ & $\mathrm{V}$ & $\mathrm{V}$ & A & $\mathrm{V}$ & A & A & A & 2 & 4 & 3 & 2 & + & - & + & - & + & + & - & + & - & Av1-3-5-6-8 \\
\hline IBCN77 & PHW1319 & 1987 & $\mathrm{~A}(\mathrm{~W})$ & A & $\mathrm{V}$ & A & A & $\mathrm{V}$ & $\mathrm{V}$ & $\mathrm{V}$ & $\mathrm{V}$ & A & $\mathrm{V}$ & A & A & A & A & 3 & 6 & 3 & 6 & + & - & - & + & + & + & + & + & - & Av1-4-5-6-7-8 \\
\hline IBCN78 & PHW1321 & 1987 & $\mathrm{~A}(\mathrm{~W})$ & A & A & $\mathrm{V}$ & A & $\mathrm{V}$ & $\mathrm{V}$ & $\mathrm{V}$ & V & $\mathrm{V}$ & A & $\mathrm{V}$ & A & A & A & 2 & 4 & 3 & 2 & + & - & + & - & + & + & - & + & - & Av1-3-5-6-8 \\
\hline IBCN79 & PHW1325 & 1987 & $\mathrm{~A}(\mathrm{~W})$ & A & A & $\mathrm{V}$ & A & $\mathrm{V}$ & $\mathrm{V}$ & $\mathrm{V}$ & V & $\mathrm{V}$ & $\mathrm{A}$ & $\mathrm{V}$ & A & A & A & 2 & 4 & 3 & 2 & + & - & + & - & + & + & - & + & - & Av1-3-5-6-8 \\
\hline IBCN80 & Leroy & 1985 & $\mathrm{C}(\mathrm{S})$ & A & A & A & A & A & $\mathrm{A}$ & A & A & $\mathrm{V}$ & A & V & A & $\mathrm{V}$ & A & 2 & 3 & 2 & 3 & + & + & + & - & + & + & - & - & + & Av1-2-3-5-6-9 \\
\hline IBCN85 & $2354-2$ & 1989 & $\mathrm{C}(\mathrm{O})$ & $\mathrm{V}$ & $\mathrm{V}$ & $\mathrm{V}$ & $\mathrm{V}$ & $\mathrm{V}$ & $\mathrm{V}$ & $\mathrm{V}$ & $\mathrm{V}$ & $\mathrm{V}$ & $\mathrm{V}$ & A & A & $\mathrm{V}$ & A & 4 & 1 & 4 & 1 & - & - & - & - & + & + & + & - & - & v-6-7 \\
\hline IBCN86 & $2362-3$ & 1989 & $\mathrm{C}(\mathrm{O})$ & A & V & A & V & V & $\mathrm{V}$ & V & V & A & V & A & A & $\mathrm{V}$ & A & 3 & 6 & 4 & 5 & - & - & - & + & + & + & + & - & - & Av4-5-6-7 \\
\hline IBCN87 & $2636-3$ & 1989 & $\mathrm{C}(\mathrm{O})$ & A & $\mathrm{V}$ & A & $\mathrm{V}$ & $\mathrm{V}$ & $\mathrm{V}$ & $\mathrm{V}$ & $\mathrm{V}$ & A & $\mathrm{V}$ & A & A & A & A & 3 & 6 & 4 & 5 & - & - & - & + & + & + & + & + & - & $7-8$ \\
\hline IBCN88 & $2365-4$ & 1989 & $\mathrm{C}(\mathrm{O})$ & A & $\mathrm{V}$ & A & V & V & V & V & V & A & V & A & A & A & A & 3 & 6 & 4 & 5 & - & - & - & + & + & + & + & + & - & Av4-5-6-7-8 \\
\hline $\mathrm{NzG}$ & $\ldots$ & ND & $\mathrm{NZ}$ & V & A & V & A & V & $\mathrm{V}$ & V & V & V & A & V & A & A & A & $\mathrm{X}$ & $X$ & 3 & 2 & - & - & + & - & + & + & - & + & - & Av3-5 \\
\hline NzT & $\ldots$ & ND & $\mathrm{NZ}$ & V & $\mathrm{V}$ & $\mathrm{V}$ & $\mathrm{V}$ & $\mathrm{V}$ & $\mathrm{V}$ & $\mathrm{V}$ & $\mathrm{V}$ & $\mathrm{V}$ & $\mathrm{V}$ & $\mathrm{V}$ & A & A & A & 4 & 1 & 4 & 1 & - & - & - & - & + & + & - & + & - & Av5-6-8 \\
\hline PHW1223 & $\ldots$ & ND & A & V & $\mathrm{V}$ & $\mathrm{V}$ & V & A & A & A & A & V & $\mathrm{V}$ & V & A & A & A & 4 & 1 & $X$ & $\mathrm{X}$ & - & - & - & - & + & + & - & + & + & Av5-6-8-9 \\
\hline PT1 & $\ldots$ & ND & $\mathrm{E}(\mathrm{Pt})$ & $\mathrm{V}$ & $\mathrm{V}$ & $\mathrm{V}$ & $\mathrm{V}$ & $\mathrm{V}$ & $\mathrm{V}$ & $\mathrm{V}$ & $\mathrm{V}$ & $\mathrm{V}$ & $\mathrm{V}$ & A & V & A & A & 4 & 1 & 4 & 1 & - & - & - & - & - & + & + & + & - & Av6-7-8 \\
\hline RAW4 & $\ldots$ & 1992 & $\mathrm{E}(\mathrm{P})$ & $\mathrm{V}$ & $\mathrm{V}$ & $\mathrm{V}$ & $\mathrm{V}$ & $\mathrm{V}$ & $\mathrm{V}$ & $\mathrm{V}$ & $\mathrm{V}$ & $\mathrm{V}$ & $\mathrm{V}$ & A & A & A & A & 4 & 1 & 4 & 1 & - & - & - & - & + & + & + & + & - & Av5- \\
\hline UK1 & $\ldots$ & 1996 & $\mathrm{E}(\mathrm{Uk})$ & $\mathrm{V}$ & $\mathrm{V}$ & $\mathrm{V}$ & V & V & $\mathrm{V}$ & $\mathrm{V}$ & $\mathrm{V}$ & $\mathrm{V}$ & $\mathrm{V}$ & A & A & A & A & 4 & 1 & 4 & 1 & - & - & - & - & + & + & + & + & - & Av5-6-7-8 \\
\hline UWA M3 & $\ldots$ & 2001 & $\mathrm{~A}(\mathrm{~W})$ & A & $\mathrm{V}$ & A & A & V & $\mathrm{V}$ & $\mathrm{V}$ & $\mathrm{V}$ & A & $\mathrm{V}$ & A & A & A & $\mathrm{V}$ & 3 & 6 & 3 & 6 & + & - & - & + & + & - & + & + & - & Av1-4-5-7-8 \\
\hline UWA P11 & $\ldots$ & 2001 & $\mathrm{~A}(\mathrm{~W})$ & A & $\mathrm{V}$ & $\mathrm{V}$ & A & $\mathrm{V}$ & $\mathrm{V}$ & $\mathrm{V}$ & $\mathrm{V}$ & $\mathrm{V}$ & $\mathrm{V}$ & A & A & $\mathrm{V}$ & A & 3 & 2 & 3 & 2 & + & - & - & - & + & + & + & - & - & Av1-5-6-7 \\
\hline UWA P16 & $\ldots$ & 2001 & $\mathrm{~A}(\mathrm{~W})$ & A & $\mathrm{V}$ & $\mathrm{V}$ & A & V & $\mathrm{V}$ & $\mathrm{V}$ & V & $\mathrm{V}$ & $\mathrm{V}$ & A & A & $\mathrm{V}$ & A & 3 & 2 & 3 & 2 & + & - & - & - & + & + & + & - & - & Av1-5-6-7 \\
\hline UWA S12 & $\ldots$ & 2002 & $\mathrm{~A}(\mathrm{~W})$ & A & $\mathrm{V}$ & A & V & V & $\mathrm{V}$ & $\mathrm{V}$ & $\mathrm{V}$ & A & $\mathrm{V}$ & A & A & A & A & 3 & 6 & 4 & 5 & - & - & - & + & + & + & + & + & - & Av4-5-6-7-8 \\
\hline UWA S7 & $\ldots$ & 2002 & $\mathrm{~A}(\mathrm{~W})$ & V & $\mathrm{V}$ & $\mathrm{V}$ & V & V & $\mathrm{V}$ & $\mathrm{V}$ & V & $\mathrm{V}$ & $\mathrm{V}$ & A & A & A & A & 4 & 1 & 4 & 1 & - & - & - & - & + & + & + & + & - & Av5-6-7-8 \\
\hline WAC4028 & $\ldots$ & 1984 & $\mathrm{~A}(\mathrm{~W})$ & V & A & V & A & V & $\mathrm{V}$ & V & V & V & A & V & A & $\mathrm{V}$ & A & $X$ & $X$ & 3 & 2 & - & - & + & - & + & + & - & - & - & Av3-5-6 \\
\hline WAC 4048 & $\ldots$ & 1984 & $\mathrm{~A}(\mathrm{~W})$ & A & $\mathrm{V}$ & V & A & V & $\mathrm{V}$ & V & V & V & V & V & A & A & A & 3 & 2 & 3 & 2 & + & - & - & - & + & + & - & + & - & Av1-5-6-8 \\
\hline WAC4078 & $\ldots$ & 1984 & $\mathrm{~A}(\mathrm{~W})$ & V & A & V & A & V & ND & V & $\mathrm{V}$ & V & A & V & A & A & A & $\mathrm{X}$ & $X$ & 3 & 2 & - & - & + & - & + & + & - & + & - & Av3-5-6-8 \\
\hline WAC4094 & $\ldots$ & 1984 & $\mathrm{~A}(\mathrm{~W})$ & A & $\mathrm{A}$ & $\mathrm{V}$ & $\mathrm{A}$ & $\mathrm{V}$ & $\mathrm{V}$ & $\mathrm{V}$ & $\mathrm{V}$ & $\mathrm{V}$ & $\mathrm{A}$ & $\mathrm{V}$ & A & A & A & 2 & 4 & 3 & 2 & + & - & + & - & + & + & - & + & - & Av1-3-5-6-8 \\
\hline WAC4110 & $\ldots$ & 1984 & $\mathrm{~A}(\mathrm{~W})$ & A & $\mathrm{V}$ & V & A & V & $\mathrm{V}$ & $\mathrm{V}$ & $\mathrm{V}$ & V & $\mathrm{V}$ & V & V & $\mathrm{V}$ & A & 3 & 2 & 3 & 2 & + & - & - & - & - & + & - & - & - & Av1-6 \\
\hline WAC7740 & $\ldots$ & 1971 & $\mathrm{~A}(\mathrm{~W})$ & A & $\mathrm{V}$ & A & $\mathrm{V}$ & $\mathrm{V}$ & $\mathrm{V}$ & $\mathrm{V}$ & $\mathrm{V}$ & A & $\mathrm{V}$ & A & A & $\mathrm{V}$ & A & 3 & 6 & 4 & 5 & - & - & - & + & + & + & + & - & - & Av4-5-6-7 \\
\hline WAC7803 & $\ldots$ & 1973 & $\mathrm{~A}(\mathrm{~W})$ & $\mathrm{V}$ & $\mathrm{V}$ & $\mathrm{V}$ & V & A & $\mathrm{A}$ & $\mathrm{A}$ & A & $\mathrm{V}$ & $\mathrm{V}$ & $\mathrm{V}$ & V & $\mathrm{V}$ & A & 4 & 1 & $X$ & $\mathrm{X}$ & - & - & - & - & - & + & - & - & + & Av6-9 \\
\hline H5 & $\ldots$ & 1990 & $\mathrm{E}(\mathrm{F})$ & $\mathrm{V}$ & $\mathrm{V}$ & $\mathrm{V}$ & V & $\mathrm{V}$ & $\mathrm{V}$ & $\mathrm{V}$ & V & $\mathrm{V}$ & $\mathrm{V}$ & A & A & A & A & 4 & 1 & 4 & 1 & - & - & - & - & + & + & + & + & - & Av5-6-7-8 \\
\hline v23.1.2 & $\mathrm{JN} 2$ & 1997 & ive & V & $\mathrm{V}$ & $\mathrm{V}$ & V & V & $\mathrm{V}$ & $\mathrm{V}$ & V & $\mathrm{V}$ & $\mathrm{V}$ & A & A & A & A & 4 & 1 & 4 & 1 & - & - & - & - & + & + & + & + & - & Av5-6-7-8 \\
\hline
\end{tabular}


and Avr loci was used to calculate genetic distances (33) between genotypes. The resulting matrix was used for a neighbor-joining cluster analysis using the "Populations" software (26). Trees were edited by using the TreeView software (34). Similarities in the race structure of populations were estimated based on Fisher's exact test on contingency tables for difference in allele frequencies using the genic differentiation option of the Genepop 3.3 software (39).

\section{RESULTS}

Pathogenicity grouping of isolates using the PG/A terminology. Isolates were classified into PG2 to PG4 and A1 to A6 groups according to their differential interaction on the differential sets 1 or 2 (Table 1). The differential set 1 allowed us to identify three PGs (PG2 to PG4) and five A groups (A1, A2, A3, A4, and A6), along with five isolates falling into an additional PG/A group (i.e., isolates that were avirulent on Glacier but not on QuintaV) (Table 1). The use of additional Rlm2 and $R \operatorname{lm} 3$ lines allowed us to show that this group corresponded mostly to isolates possessing AvrLm3, recognized by Rlm3 present in Glacier (IBCN55, Nz-G, WAC4028, and WAC4078) (Table 1). With the differential set 2 , three isolates corresponded to such new PG/A groups. In all, 15 isolates were inconsistently classified when using either differential 1 or differential 2. These discrepancies mainly were found to be due to (i) the presence of $R \operatorname{lm} 4$ in the QuintaV line used here $(9,13)$, which led to the classification of seven AvrLm4 isolates into PG3-A6, instead of PG4-A5; this was illustrated by isolate IBCN05 from Germany; or (ii) the presence of both Rlm2 and Rlm3 in Glacier (9), which led to the classification of seven AvrLm3 isolates into an undescribed PG, PG2-A4 (differential set 1), or PG3-A2 (differential set 2); this was the case for isolates IBCN78 and IBCN79, for which genetic analyses demonstrated they actually possessed both AvrLm1 and AvrLm3 alleles (9). Additionally, the PG4-A1 reference isolate PHW1223 was classified as PG4-A1 with the differential set 1 but fell into a new PG/A group with the differential set 2. The use of additional differential lines and genetic analyses involving this isolate showed that it harbored AvrLm9, recognizing RIm9 present in Bristol (Table 1) (described below).

Classification of isolates into PGs A1 to A6 clearly indicated major differences among the populations from the three continents, whatever the differential used (Fig. 1). However, inconstancies between results from the two differential sets, as well as the occurrence of isolates corresponding to unknown groups, indicated that the analyses of the race structure of $L$. maculans populations can be based only on the knowledge of Avr alleles for each Avr locus described to date. For this purpose, it first was necessary to elucidate the genetic control of specific interaction on Darmor (13) and to improve our knowledge of the $R$ genes present in the differentials.

Evidence for new specific interactions: resistance in cvs. Darmor, Goéland, and Mohican. Delourme et al. (13) described a new specific interaction between IBCN56 and Darmor. One major gene, termed $R \operatorname{lm} 9$, was identified in Darmor and was suggested to recognize the putative Avr gene AvrLm9 in IBCN56. IBCN56 was used here to screen 29 winter-type $B$. napus cultivars for the presence of $R \operatorname{lm} 9$. The presence of $R \operatorname{lm} 1$ to $R \operatorname{lm} 8$ in these cultivars also was analyzed using the reference isolates v11.1.1, v11.1.2, v23.2.1, 22.2.2, and IBCN78 or $\operatorname{IBCN79}(9,13)$ (Table 1). In addition to Darmor, two winter-type cultivars (Goéland and Mohican) were found both to be resistant to isolate IBCN56 and to lack Rlm1 to Rlm8 (Tables 1 and 2; data not shown). This suggested the occurrence of $R \operatorname{lm} 9$ alone in these three cultivars. Thus, the whole collection of isolates was characterized on Darmor, Goéland, and Mohican. Twelve isolates of the collection, namely IBCN13, IBCN51-59, IBCN80, PHW1223, and WAC7803, behaved as IBCN56, inducing a complete resistance on all three cvs. Darmor, Goéland, and Mohican (Table 1; data not shown).

Genetic analysis of resistance on Darmor, Goéland, and Mohican. Two crosses involving avirulent isolates on Darmor were performed. In cross 58, PHW1223 was crossed with v23.1.2 (Table 1) and the progeny (46 isolates) was inoculated on Darmor, Goéland, Mohican (putative Rlm9 cultivars), 22-1-1 (Rlm3, susceptible control in this cross), 23-2-1 (Rlm7), and Bristol $(R \operatorname{lm} 2)$ because PHW1223 was found to be avirulent on Bristol

TABLE 2. List and characteristics of cultivars and lines used to characterize the collection of isolates of Leptosphaeria maculans

\begin{tabular}{|c|c|c|c|c|c|}
\hline \multirow[b]{2}{*}{ Plant genotype } & \multirow[b]{2}{*}{ Known resistance gene } & \multirow[b]{2}{*}{ Reference } & \multicolumn{3}{|c|}{ Additional information resulting from this work ${ }^{\mathrm{a}}$} \\
\hline & & & $\operatorname{Rlm} 9$ & $R \operatorname{lm} W$ & $R \operatorname{lm} X$ \\
\hline Westar & None & 40 & - & - & - \\
\hline \multicolumn{6}{|l|}{ Differential set 1} \\
\hline Glacier & $R \operatorname{lm} 2+R \operatorname{lm} 3$ & 9 & - & - & - \\
\hline QuintaV & $R \operatorname{lm} 1+R \operatorname{lm} 4$ & $8,9,13$ & - & - & - \\
\hline Jet Neuf & $R \operatorname{lm} 4$ & 8 & - & - & - \\
\hline \multicolumn{6}{|l|}{ Differential set 2} \\
\hline Columbus & $R \operatorname{lm} 1+R \operatorname{lm} 3$ & 9 & - & - & - \\
\hline Bristol & $R \operatorname{lm} 2$ & 9 & + & - & - \\
\hline \multicolumn{6}{|l|}{ Other differentials } \\
\hline $04-1-2$ & $R \operatorname{lm} 2+R \operatorname{lm} 7$ & 9, unpublished data & - & - & - \\
\hline $148-1-1$ & $R \operatorname{lm} 7$ & 9 & - & - & - \\
\hline $149-2-1$ & $R \operatorname{lm} 1+R \operatorname{lm} 7$ & 9 & - & - & - \\
\hline $150-2-1 \& 151-2-1$ & $R \operatorname{lm} 5$ & 9 & - & - & - \\
\hline $156-2-1$ & $R \operatorname{lm} 8$ & 9 & - & - & - \\
\hline $22-1-1$ & $R \operatorname{lm} 3$ & 9 & - & - & - \\
\hline $23-1-1 \& 05-1-1$ & $R \operatorname{lm} 7$ & 9,13 & - & - & - \\
\hline Brassica juncea $\mathrm{cv}$. Picra & $R \operatorname{lm} 5+R \operatorname{lm} 6$ & 9 & ND & ND & ND \\
\hline Darmor & $\operatorname{Rlm} 9$ & 13 & + & + & - \\
\hline Darmor-MX & $R \operatorname{lm} 6$ & Unpublished data & ND & ND & ND \\
\hline Falcon & $R \operatorname{lm} 4$ & 40,42 & - & - & - \\
\hline Falcon-MX & $R \operatorname{lm} 6+R \operatorname{lm} 4$ & 9 & - & - & - \\
\hline Goéland & $\ldots$ & 40,42 & + & + & - \\
\hline Mohican & $\ldots$ & 40,42 & + & - & - \\
\hline Navajo & $R \operatorname{lm} 3$ & 42, unpublished data & - & - & - \\
\hline Pollen & $R \operatorname{lm} 4$ & 40 & - & - & - \\
\hline Samouraï & $R \operatorname{lm} 2$ & 13,40 & + & + & + \\
\hline Samourai-MX & $R \operatorname{lm} 6+R \operatorname{lm} 1$ & Unpublished data & ND & ND & ND \\
\hline
\end{tabular}

a Symbols: $-=$ absence of the resistance $(R)$ gene, $+=$ presence of the $R$ gene, $\mathrm{ND}=$ not determined. 
although being virulent on Glacier (Rlm2 and $R \operatorname{lm} 3$ ) (Table 1). When inoculated on Darmor, the progeny isolates displayed interaction phenotypes either clearly avirulent (mean disease rating $<3,18$ isolates) or virulent (mean disease rating $>4,28$ isolates) (Fig. 2). This 18:28 A:V segregating ratio fitted a single gene control of avirulence $\left(\chi^{2}=2.174, P=0.1404\right)$, suggesting the occurrence of an Avr gene in PHW1223, termed AvrLm9, recognizing $\operatorname{Rlm} 9$ in Darmor. Isolates of the progeny behaved similarly on Darmor, Goéland, and Bristol, suggesting that these three cultivars all possessed $R \operatorname{lm} 9$. Segregation on Mohican was more complicated, with a continuous distribution of mean disease rating between that of the two parental isolates (Fig. 2). This could be due to either a polygenic control of avirulence of PHW1223 on
Mohican, or a less efficient expression of $R \operatorname{lm} 9$ on Mohican, or interactions with segregation of aggressiveness, along with $A v r$ genes, in cross 58. Whatever the hypothesis, segregation on this line was not analyzed further. AvrLm7 also segregated in this cross and three classes of isolates were identified in the progeny (i.e., AvrLm7avrLm9 as the parental isolate v23.1.2, 19 isolates; avrLm7AvrLm9 as the parental isolate PHW1223, 18 isolates; and avrLm7avrLm9, 9 recombinant isolates). These data indicate that AvrLm7 and AvrLm9 are not allelic, but not independent $\left(\chi^{2}=\right.$ 20.6, df $=3, P<0.001$ ), with a genetic distance between AvrLm9 and $A v r L m 7$ estimated to be $22.6 \mathrm{cM}$. The lack of recovery of the second recombinant class, AvrLm7AvrLm9, could be due to the limited number of progeny analyzed.
A

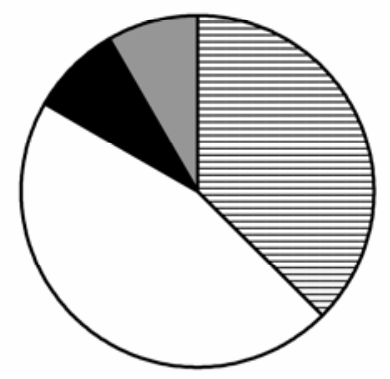

B

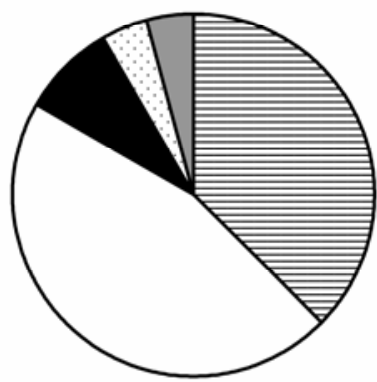

Canada
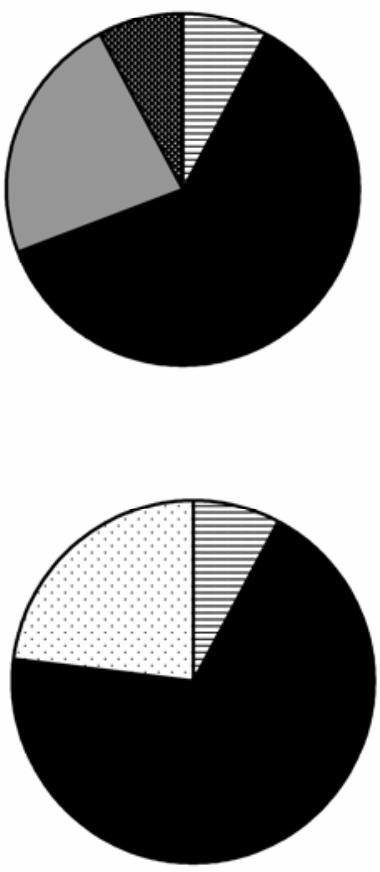

Australia
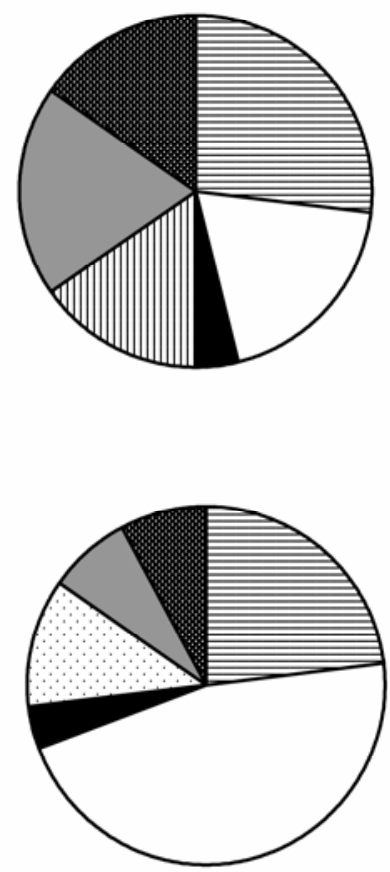

Fig. 1. Distribution of pathogenicity groups A1 to A6 of Leptosphaeria maculans in Europe, Canada, or Australia. Grouping based on differential interactions on A, cvs. Westar, QuintaV, Glacier, and Jet Neuf (differential set 1) and B, cvs. Westar, Columbus, Bristol, and Jet Neuf (differential set 2). Horizontal bars, group A1; white, A2; black, A3; vertical bars, A4; black points on white background, A5; gray, A6; white points on black background, X (isolates belonging to still undescribed groups).

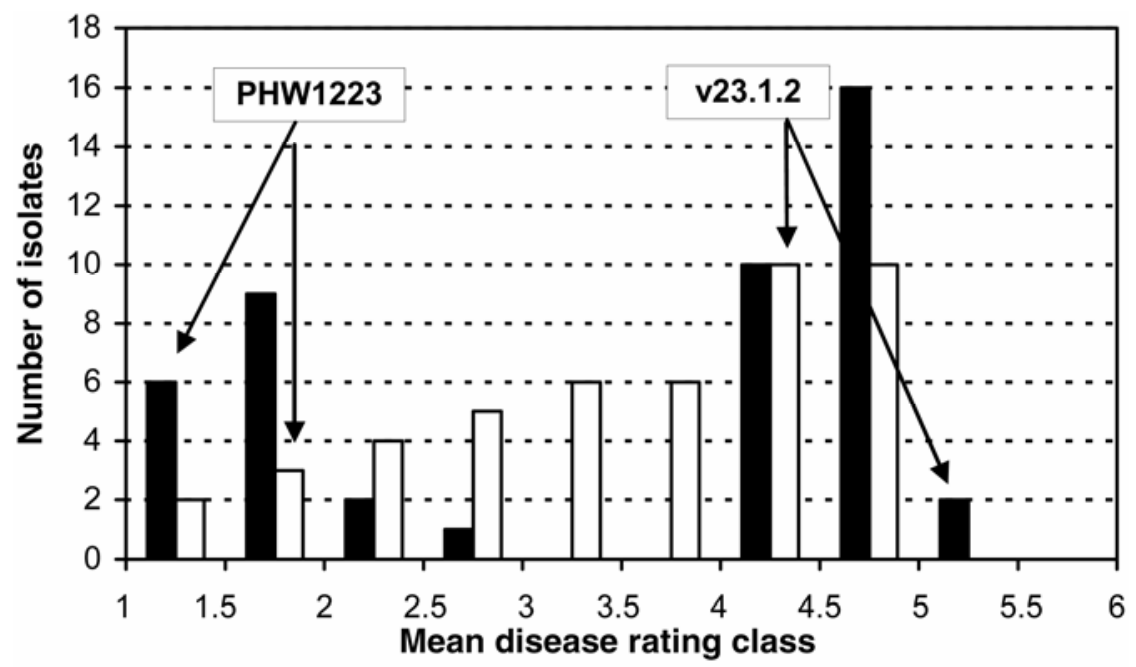

Fig. 2. Frequency distribution of mean disease rating on cvs. Darmor (black) and Mohican (white) on a scale of 1 (resistant) to 6 (susceptible), in the progeny of cross 58 (PHW1223 $\times$ v23.1.2). The position of the mean disease rating of parental isolates is indicated by the arrows. 
Genetic analysis of AvrLm 9 also was performed in the independent cross 39, where IBCN59 was crossed with H5 (Table $1)$. The 61 progeny isolates recovered were analyzed on a range of $R \operatorname{lm} 2, R \operatorname{lm} 3, R \operatorname{lm} 7$, and $R \operatorname{lm} 9$ cultivars (Table 3 ). Progeny isolates behaved always identically (i.e., either virulent or avirulent) on Goéland and Darmor. AvrLm3, AvrLm7, and avirulence on Goéland and Darmor segregated as single genes in this cross (Table 3), suggesting the occurrence of AvrLm3 and $a v r L m 7$, along with AvrLm9, in IBCN59. As reported for other crosses (9), no recombinant isolates between AvrLm3 and AvrLm7 were detected in this progeny. In contrast, as in cross 58 , recombinant isolates between AvrLm 7 and AvrLm9 were detected (i.e., AvrLm7AvrLm9 or avrLm7avrLm9 isolates), with a 20:30:2:9 ratio for avrLm7AvrLm9:AvrLm7avrLm9:AvrLm7AvrLm9:avrLm7avrLm9 isolates (data not shown). These data confirmed results from cross 58, indicating a genetic linkage between AvrLm7 and AvrLm9 $(P<0.001)$, with a genetic distance $(22.4 \mathrm{cM})$ similar to that found in cross 58. The 39:22 A:V segregation on Bristol fitted a two-independent-gene control $\left(\chi^{2}=3.94, P=0.0459\right)$ better than a single-gene control $\left(\chi^{2}=4.738, P=0.0295\right)$. A two-gene control also was found for avirulence on Samouraï. Because analysis of cross 58 indicated the presence of $R \operatorname{lm} 9$ in Bristol, the simplest hypothesis to explain these data is that IBCN59 also possessed AvrLm2, recognizing Rlm2 in Bristol. This hypothesis is supported by the fact that, from the 37 avrLm 9 progeny isolates, 17 and 20 were avirulent and virulent, respectively, on Bristol, which fits a 50:50 ratio $\left(\chi^{2}=0.243, P=0.622\right)$. However, in contrast to cross 58 , cross 39 revealed an additional level of complexity, because four isolates of the progeny displayed interaction phenotypes that could not be explained by the currently identified Avr$R$ gene interactions (Table 4 ). Two isolates were found to be viru- lent on Goéland, Darmor, and Bristol (thus identified as avrLm2avrLm9), but avirulent on Samouraï, suggesting the occurrence of an additional $R$ gene in this last cultivar, temporarily termed $R \operatorname{lm} X$. Similarly, two isolates were found to be virulent on Bristol and thus genotyped avrLm2avrLm9, but were avirulent on Goéland, Darmor, and Samouraï, suggesting again the occurrence of an unknown, additional $R$ gene (termed $R \operatorname{lm} W$ ) in these last three cultivars (Table 4).

Isolates PHW1223, IBCN59, or recombinant isolates from cross 39 were used to identify the presence of $R \operatorname{lm} 9, R \operatorname{lm} W$, and $R \operatorname{lm} X$ in the differential lines (Table 2). For instance, the virulence of IBCN59 on lines 23-1-1, 156-2-1, and Pollen was sufficient to conclude that these three lines did not possess $R \operatorname{lm} 9, R \operatorname{lm} W$, or $R \operatorname{lm} X$ (Table 2).

Strategy for the identification of Avr alleles in the collection. Due to the identification of $R \operatorname{lm} 9, R \operatorname{lm} W$, and $R \operatorname{lm} X$ in some lines of the differential, or to the impossibility to determine the presence of these genes in some lines (Table 2), identification of Avr alleles was done mostly in two steps. The use of lines Westar, QuintaV, Columbus, Bristol, 22-1-1, Jet Neuf, 150-2-1, DarmorMX, 23-1-1, 156-2-1, and Darmor often was sufficient to characterize Avr alleles for all loci, particularly for isolates virulent on Darmor or isolates with a limited number of Avr alleles. However, the results always were confirmed by using a second set of lines as described above (for instance, Samouraï, Navajo, Pollen or Falcon, 151-2-1, 148-1-1, Goéland, and Mohican). Isolates avirulent on Darmor were classified AvrLm9 whenever they were consistently avirulent on Goéland, Bristol, and Samouraï. For AvrLm9 isolates, identification of other Avr alleles was (i) possible for AvrLm6 only in the case of avrLm4 isolates, by using the Rlm6 line Falcon-MX; and (ii) difficult for AvrLm2, because Bristol and

TABLE 3. Segregation analysis of avirulence of Leptosphaeria maculans toward a range of Brassica napus cultivars or lines in cross 39 (IBCN59 $\times$ H5) ${ }^{\mathrm{a}}$

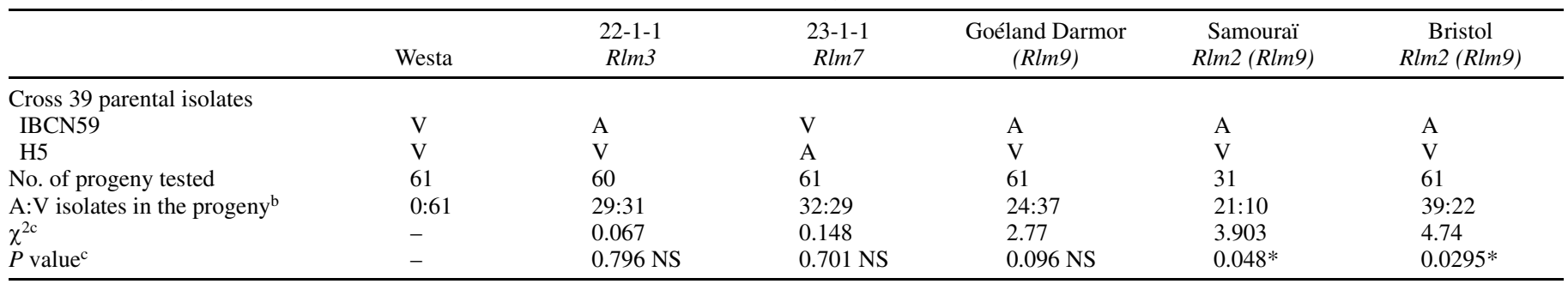

${ }^{\text {a }}$ Resistance $(R)$ genes in parentheses are putative $R$ genes deduced from the present study; $\mathrm{A}=$ the isolate is avirulent; $\mathrm{V}=$ the isolate is virulent.

${ }^{\mathrm{b}}$ Values given are number of isolates in each phenotypic class $\mathrm{A}$ (avirulent) or $\mathrm{V}$ (virulent).

${ }^{\mathrm{c}} \chi^{2}$ and $P$ values for the single gene control hypothesis (50:50 A:V ratio). NS $=$ the observed ratio is not significantly different from the tested hypothesis at a 5\% level; $*=$ the ratio is significantly different at the $5 \%$ level $;-=$ the interaction phenotype on this line or cultivar is not segregating in this cross

TABLE 4. Differential isolates of Leptosphaeria maculans, identified in the progeny of cross 39 (IBCN59 $\times$ H5) or in the field isolate collection, inoculated on cvs. Darmor, Goéland, Bristol, and Samouraï

\begin{tabular}{|c|c|c|c|c|c|c|}
\hline \multirow[b]{2}{*}{ Cross 39} & \multicolumn{4}{|c|}{ Differential Brassica napus cultivars ${ }^{\mathrm{a}}$} & \multirow[b]{2}{*}{ Putative isolate genotype $\mathrm{b}^{\mathrm{b}}$} & \multirow[b]{2}{*}{ Other field isolates ${ }^{\mathrm{c}}$} \\
\hline & $\begin{array}{l}\text { Goéland } \\
R \operatorname{lm} 9 \\
(R \operatorname{lm} W)\end{array}$ & $\begin{array}{l}\text { Darmor } \\
R \operatorname{lm} 9 \\
(R \operatorname{lm} W)\end{array}$ & $\begin{array}{l}\text { Bristol } \\
\operatorname{Rlm} 2 \\
\operatorname{Rlm} 9\end{array}$ & $\begin{array}{c}\text { Samourä̈ } \\
R \operatorname{lm} 2 \operatorname{Rlm} 9 \\
(R \operatorname{lm} W)(R \operatorname{lm} X)\end{array}$ & & \\
\hline IBCN59 & A & A & A & A & $A 2 A 9 A W A X$ & $\ldots$ \\
\hline $\mathrm{H} 5$ & V & V & V & V & $a 2 a 9 a W a X$ & $\ldots$ \\
\hline Explained phen & & & & & & \\
\hline \multirow{3}{*}{$\begin{array}{l}\text { Unexplained phenotypes in progeny } \\
\text { (Two isolates) } \\
\text { (Two isolates) }\end{array}$} & & & & & & \\
\hline & A & A & $\mathrm{V}$ & A & $a 2 \mathrm{a} 9 A W ?$ & None \\
\hline & V & V & V & A & $a 2 \mathrm{a} 9 a W A X$ & IBCN68 \\
\hline
\end{tabular}


Samouraï possessed $\operatorname{Rlm} 9$. In this case, however, it was possible to conclude for the presence of AvrLm2 whenever isolates were avirulent on Glacier (Rlm2 and Rlm3) but virulent on 22-1-1 $(R \operatorname{lm} 3)$, such as IBCN13, or avirulent on $04-1-2(R \operatorname{lm} 2$ to $R \operatorname{lm} 7)$ but virulent on 23-1-1 (Rlm7), such as IBCN52-59 (Table 1; data not shown).

Avr allele frequencies in the collection. Almost all isolates could be genotyped for AvrLml-AvrLm9 (Table 1). The only exceptions regarded two Canadian isolates harboring AvrLm4 and AvrLm9, for which the presence of AvrLm6 could not be determined (Table 1). Depending on the Avr locus and the geographic origin of the isolate, the frequency of the Avr alleles ranged from 0 (AvrLm3 and AvrLm9 in Europe) to 1 (AvrLm6 and AvrLm7 in Europe) (Table 5). For some loci, the frequencies were not very different from one continent to the other. This was the case for AvrLml (46.1 to 61.5\%), AvrLm4 (16.7 to 38.5\%), AvrLm5 (88.5 to $100 \%$ ), and AvrLm6 (88.5 to $100 \%)$. In contrast, major and significant differences were observed between continents for AvrLm2, AvrLm3, AvrLm7, AvrLm8, and AvrLm9 (Table 5).

Proposal for a new race terminology. A race of a pathogen is identified by its pattern of avirulence alleles; therefore, we propose to term each L. maculans race by the successive numbers of Avr loci for which the isolate has been characterized and is avirulent, preceded by the letters "Av", to show that only Avr alleles are indicated. For instance, race Av1-2-4-7 is composed of isolates possessing the Avr alleles AvrLm1, AvrLm2, AvrLm4, and AvrLm7. In addition, we also propose to indicate in parentheses the Avr loci for which the genotyping has not been possible; for instance, Av1-2-3-4-5-9-(6) for isolate IBCN53, for which AvrLm6 has not been determined. This isolate can belong to either race Av1-2-3-4-5-9 or race Av1-2-3-4-5-6-9.

Race structure of the collection. Whatever the genotype of isolates IBCN53 and IBCN54 at the AvrLm6 locus, their combination of Avr alleles for all other loci is unique in the collection; therefore, they belong to one race distinct from all others identified in this collection. Therefore, a minimum number of 26 different races of the pathogen were identified in the collection (Tables 5 and 6). These 26 races were characterized by the combination of two to eight virulences, and were represented by one to nine isolates in the collection (Table 6). Thirteen races were represented by only one isolate and, in most cases, these isolates originated from Australia (9 of 13). From the 13 races that were represented by more than one isolate, 7 comprised isolates from two different continents (Table 6) but only 1 race was detected in the three continents (Av4-5-6-7-8). The two races represented by the highest number of isolates were composed mainly of European isolates (i.e., race Av1-5-6-7-8 [only European isolates] and race Av5-6-7-8 [88.9\% of the isolates of this race being from Europe]). This reflects the lower diversity detected in isolates sampled in Europe ( 8 races for 24 isolates) compared with that identified in Australia (18 races for 24 isolates).

Another major difference between samplings from Europe and Australia, for which a similar and sufficient number of isolates was available, concerned the number of virulence alleles per isolate (Fig. 3). The mean number of virulence alleles per isolate de- creased from 5.11 in Australia, to 4.33 in Europe and 3.46 in Canada. Although the majority of isolates possessed five virulence alleles in both Europe and Australia, the more simple races (few virulences) were identified in Canada and Europe, whereas the most complex races (many virulences) were found in Australia (Fig. 3). There is no relationship between the year of sampling and the number of virulence per isolate. It has to be noticed that the two oldest European isolates, BBA62908 and IBCN74, were the two isolates possessing the highest number of Avr alleles; in contrast, one of the isolates possessing the lowest number of Avr alleles, WAC7803, was isolated in Australia in 1973, on R. raphanistrum (Table 1).

Finally, distance analyses based on Avr alleles revealed three clear-cut clusters of isolates (Fig. 4). Mainly, Australian isolates were found in all branches of the dendrogram. European isolates were found in only two branches. The two New Zealand isolates were clustered with Australian isolates. Canadian isolates were split into two groups, with isolates from Ontario being close to European isolates, whereas isolates from Western Canada were included in the main Australian cluster (Fig. 4).

Comparison between race structure and PG/A classification. Each of the three pathogenicity groups PG2 to PG4 contained from 5 to 10 distinct races of the pathogen, depending on

TABLE 6. Races of Leptosphaeria maculans identified in the collection

\begin{tabular}{|c|c|c|c|}
\hline Race $^{\mathrm{a}}$ & No. of isolates & Origin $^{b}$ & No. of virulence \\
\hline Av1-4-5-7-8 & 1 & A & 4 \\
\hline Av2-3-5-6-9 & 1 & $\mathrm{C}$ & 4 \\
\hline Av1-2-4-7 & 1 & A & 5 \\
\hline Av5-6-8-9 & 1 & A & 5 \\
\hline Av2-5-6-9 & 1 & A & 5 \\
\hline Av1-6-7-8 & 1 & $\mathrm{E}$ & 5 \\
\hline Av3-5-6 & 1 & A & 6 \\
\hline Av5-6-7 & 1 & $\mathrm{C}$ & 6 \\
\hline Av6-7-8 & 1 & $\mathrm{E}$ & 6 \\
\hline Av1-6 & 1 & A & 7 \\
\hline Av5-6 & 1 & A & 7 \\
\hline Av6-9 & 1 & $\mathrm{~A}$ & 7 \\
\hline Av5 & 1 & A & 8 \\
\hline Av1-2-3-4-5-9-(6) & 2 & $\mathrm{C}$ & $2-3^{c}$ \\
\hline Av1-2-4-5-6-7-8 & 2 & $\mathrm{E}$ & 2 \\
\hline Av1-4-5-6-7-8 & 2 & $\mathrm{~A}, \mathrm{E}$ & 3 \\
\hline Av1-5-6-8 & 2 & A & 5 \\
\hline Av4-5-6-7 & 2 & $\mathrm{~A}, \mathrm{C}$ & 5 \\
\hline Av3-5-6-8 & 2 & A, NZ & 5 \\
\hline Av5-6-8 & 2 & A, NZ & 6 \\
\hline Av1-3-5-6-8 & 4 & A & 4 \\
\hline Av1-5-6-7 & 4 & $\mathrm{E}, \mathrm{A}$ & 5 \\
\hline Av1-2-3-5-6-9 & 5 & $\mathrm{C}$ & 3 \\
\hline Av4-5-6-7-8 & 5 & $\mathrm{~A}, \mathrm{C}, \mathrm{E}$ & 4 \\
\hline Av1-5-6-7-8 & 8 & $\mathrm{E}$ & 4 \\
\hline Av5-6-7-8 & 9 & A (1 isolate), E & 5 \\
\hline
\end{tabular}

a Race nomenclature indicates the avirulence (Avr) loci for which the isolate is avirulent. Numbers in parentheses correspond to Avr alleles not characterized.

b $\mathrm{A}=$ Australia, $\mathrm{C}=$ Canada, $\mathrm{E}=$ Europe , and $\mathrm{NZ}=$ New Zealand.

$c$ The number of virulence depends on the data for the missing genotype.

TABLE 5. Number of races and frequencies of avirulence (Avr) alleles in the collection of Leptosphaeria maculans

\begin{tabular}{|c|c|c|c|c|c|c|c|c|c|c|c|}
\hline \multirow[b]{2}{*}{ Geographic origin } & \multicolumn{2}{|c|}{ Number of } & \multicolumn{9}{|c|}{ Frequency of Avr alleles } \\
\hline & Isolates & Races & AvrLm1 & AvrLm2 & AvrLm3 & AvrLm4 & AvrLm5 & AvrLm6 & AvrLm7 & AvrLm8 & AvrLm9 \\
\hline Europe & 24 & 8 & 0.583 & 0.083 & 0.00 & 0.167 & 0.917 & 1.00 & 1.00 & 0.917 & 0.00 \\
\hline Canada & 13 & 6 & 0.615 & 0.692 & 0.692 & 0.385 & 1.00 & 1.00 & 0.308 & 0.154 & 0.692 \\
\hline Australia $^{a}$ & 26 & 18 & 0.462 & 0.077 & 0.269 & 0.231 & 0.885 & 0.885 & 0.346 & 0.615 & 0.115 \\
\hline Total $^{\mathrm{b}}$ & 63 & 26 & 0.540 & 0.206 & 0.254 & 0.238 & 0.921 & 0.951 & 0.587 & 0.635 & 0.177 \\
\hline$P$ values ${ }^{c}$ & $\ldots$ & $\ldots$ & 0.9402 & 0.0006 & $<0.0001$ & 0.7659 & 0.833 & 0.2099 & $<0.0001$ & 0.0001 & $<0.0001$ \\
\hline
\end{tabular}

a Including the two isolates from New Zealand.

b The whole collection.

c $P$ values for the contingency tests for differences in allele frequencies across the three populations (Fisher's exact test). 
the PG and the differential set used (Table 7). Similarly, except for the A4 group identified with the differential set 1, composed of one single race, all other A groups were composed of more than one race (data not shown). A minimum of five distinct Avr loci could be found to be polymorphic between two isolates belonging to the same PG (Table 7). In particular, all Avr loci were polymorphic between PG2 isolates identified with the differential set 2. It has to be noticed that the two PG2 reference isolates, IBCN80 from Canada (23) and IBCN74 from France (5,7,35), displayed polymorphism for five Avr loci, namely AvrLm3, AvrLm4, AvrLm7, AvrLm8, and AvrLm9 (Table 1). As a consequence, they belong to very distant clusters (Fig. 4). PG4-A1 reference isolates H5 (5) and IBCN01 $(7,23,46)$, both from Europe, displayed the same Avr allele composition but differed from the PG4 reference isolate PHW1223 from Australia, $(4,35)$ at the two loci AvrLm7 and AvrLm9 (Table 1). Except for A4 group, all other A1 to A6 groups also displayed at least one polymorphic Avr locus, with a maximum of seven and eight polymorphic Avr loci for isolates belonging to the A3 group identified with the differential sets 1 and 2, respectively (data not shown).

\section{DISCUSSION}

Knowledge of race structure of $L$. maculans at a worldwide scale has major implications in terms of choice of sources of major $R$ genes to deploy, possible transfer of resistance sources between regions, or potential risks to disseminate new races from one continent to another. At present, detailed studies on the virulence pattern of populations of L. maculans have been performed in a few countries only (e.g., Germany [24], France [40], Canada $[25,30,32]$, and Australia [19,20,32]). None of these studies allows any comparison between race structure in different countries or continents, mainly because they lacked a common race terminology or standard plant and isolate differentials. The IBCN collection was built to give access to the scientific community to a common set of isolates of $L$. maculans collected worldwide (41). Since its existence in 1994, the IBCN collection has been analyzed widely at a molecular level, allowing major progress in

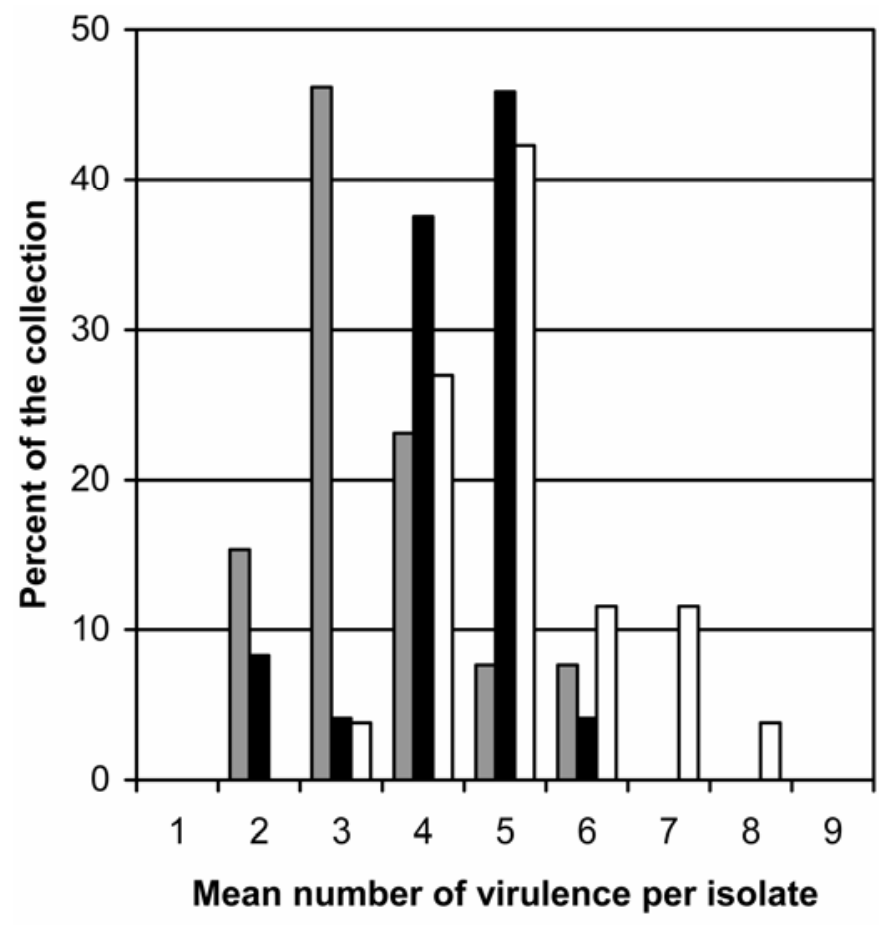

Fig. 3. Frequency distribution of the number of virulence alleles per isolate for Leptosphaeria maculans collections from Australia (white), Canada (gray), and Europe (black). identifying the main components of the " $L$. maculans species complex" and in the development of molecular tools to identify or analyze each component of the complex $(22,31,37)$. To date, however, only one report describes interaction phenotypes of IBCN isolates, using the differential set 1 , leading to a classification of isolates into A1 to A6 groups (46). The present study is the first attempt to genotype isolates of a worldwide collection of L. maculans, including the IBCN isolates, at all described Avr loci.

This work first points out the inadequacy of the PG/A terminology, illustrated by the existence of isolates that do not fit in any of the known PG/A groups, as already reported $(5,30,36)$, or by the discrepancies between classifications, depending on the differential set used. In addition to the problem of heterogeneity of the plant genotypes Quinta and Glacier $(8,23)$, the main problem was found here to be due to the frequent occurrence of more than one $R$ gene in each differential line. Previous studies described the occurrence of both $R \operatorname{lm} 1$ and $R \operatorname{lm} 4$ in Quinta $(8,13), R \operatorname{lm} 2$ and $R \operatorname{lm} 3$ in Glacier (9), or Rlm1 and Rlm3 in Columbus (9). Columbus was chosen to replace Quinta in the differential set, because it was commercially available and marketed as a pure line. Similarly, Bristol (Rlm2) was selected as a differential to replace, as a pure line, Glacier $(40,42)$. However Bristol also was found here to possess $R \operatorname{lm} 9$, making it impossible to classify some PG4 isolates into any described PG/A group when using Bristol as a differential, because of the presence of AvrLm 9 in these isolates.

The present study demonstrated that, without the current availability of differential lines each possessing only a single $R$ gene, the genotyping of isolates necessitates the use of a wide range of lines with complementary combinations of $R$ genes, especially for isolates displaying numerous Avr alleles. The accuracy of the resulting genotyping was supported here by genetic analyses; as, for instance, in the cross involving IBCN59, in which AvrLm2, AvrLm3, AvrLm7, and AvrLm9 actually were found to segregate as single genes in the progeny. In addition, this genotyping is fully consistent with virulence patterns of some isolates, described in previous, independent studies. For instance, the low virulence of the four Australian isolates UWAS12, UWAM3, IBCN17 (C13), and IBCN18 (M1) on cotyledons of B. napus cv. Dunkeld, compared with their virulence on Westar $(20,38)$, is fully consistent with the presence of Rlm4 in cv. Dunkeld (42) and the presence of AvrLm4 in UWAS12, UWAM3, IBCN17, and IBCN18 (this study).

Numerous distinct races may be classified in the same group when using the PG/A terminology, with up to 10 distinct races classified into the same group. This result illustrates the need for a more accurate system for race designation. The race terminology proposed here is based on knowledge of the Avr allele pattern in the isolates, and can indicate when data are missing at specific Avr loci. Therefore, it provides direct information on $R$ genes that may be used to control the disease in specific situations where the population structure has been monitored in detail. It also is a potentially evolving system, because it can be amended by taking into account any new $A v r-R$ interaction to be discovered in the future. The improved knowledge given by this terminology was illustrated here for PG2 isolates, which are described in the literature as low-virulence isolates (e.g., in Canada [25,32]), compared with European and Australian ones (25). Two PG2 reference isolates, IBCN74 from Europe $(4,7,35)$ and IBCN80 from Canada (23), actually were classified as PG2-A3 in the present study. However, they differed for five of their Avr alleles. Isolate IBCN80 is virulent at the Avr locus AvrLm7 and may represent a threat to European countries, where almost all isolates are AvrLm7 (45; this study, T. Rouxel and M. H. Balesdent, unpublished data). This shows the need to identify alleles at all loci before conferring low or high virulence status on the isolates present in one location. Finally, data indicating a prevalence of PG2 in Eastern Canada (32) with a recent surge of PG3 in Manitoba (14), 
will be useful only for disease management when the Avr locus for which this recent evolution has occurred is identified.

At present, there is an insufficient knowledge of L. maculans race structure, even as defined using the PG/A classification, in the main oilseed rape (OSR)-growing areas. The present study, although based on the analysis of a limited number of isolates, gives a first comparative overview of $L$. maculans race structure. It mainly points out unexpected major disparities for race structure among Australia, Europe, and North America. Unweighted pair-group method with arithmetic means cluster analysis of the IBCN collection with amplified fragment length polymorphism
TABLE 7. Race composition in each pathogenicity group (PG) of Leptosphaeria maculans $^{\mathrm{a}}$

\begin{tabular}{lcc}
\hline PG $^{\text {b }}$ & No. of races & $\begin{array}{l}\text { No. of polymorphic avirulence loci } \\
\text { between isolates from the same PG }\end{array}$ \\
\hline PG2 & $5(5)$ & $8(9)$ \\
PG3 & $9(10)$ & $6(7)$ \\
PG4 & $8(8)$ & $5(5)$ \\
X & $4(3)$ & $4(4)$ \\
\hline
\end{tabular}

a Values are from the classification into PGs using differential set 1 , and values in parentheses use differential set 2.

b $\mathrm{X}=$ isolates belonging to still undescribed groups.

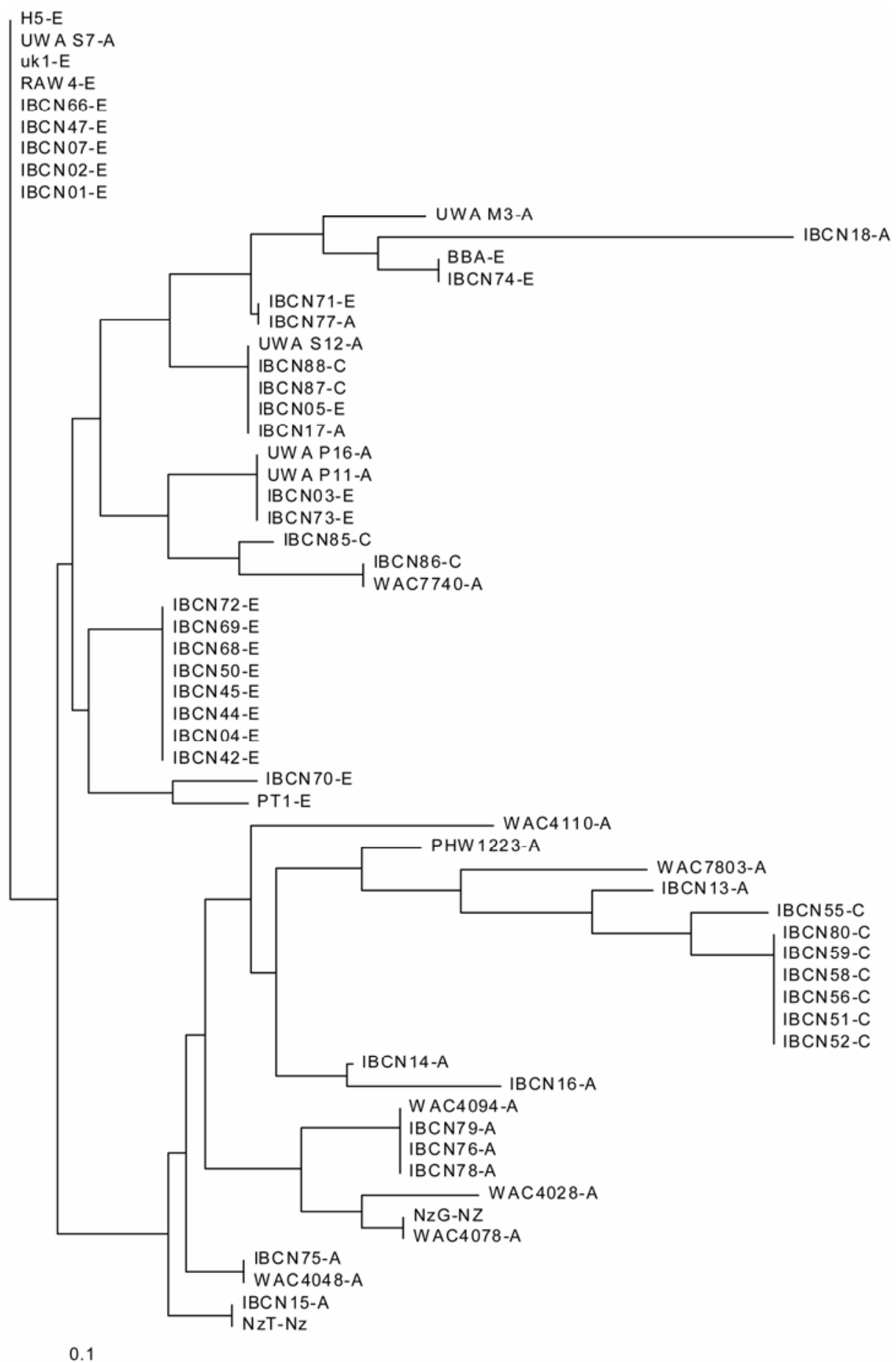

Fig. 4. Dendrogram generated by neighbor-joining analysis based on avirulence (Avr) alleles data (AvrLm1 to AvrLm9). Isolate numbers have a suffix letter referring to their geographic origin $(\mathrm{A}=$ Australia, $\mathrm{C}=$ Canada, $\mathrm{E}=$ Europe, and $\mathrm{NZ}=\mathrm{New}$ Zealand). The two isolates for which AvrLm6 genotype is missing are not represented on this tree. 
markers failed to uncover any specific population structure linked with host plant or geographic origin (37). In contrast, combination of Avr alleles clearly separates most European isolates from most Australian ones, whereas Canadian isolates tend to be more related to either European isolates (isolates from Ontario) or Australian isolates (Saskatchewan isolates). This result strongly suggests that the selection pressures exerted in different parts of the world at Avr loci may have structured the populations. European isolates analyzed here were extremely monomorph, containing only eight distinct races with two major ones, Av5-6-7-8 and Av1-5-6-7-8. All European isolates shared the absence of AvrLm3 and AvrLm9 and the constant presence of AvrLm6 and AvrLm7. Except for the two most ancient isolates analyzed here, all European isolates also lack AvrLm2. These data are consistent with what has been described for European isolates $(24,40,46)$, whose populations were strongly structured by the sequential use of resistance genes $R \operatorname{lm} 2, R \operatorname{lm} 4$, and then Rlm1, leading to the diminution or complete extinction of the corresponding Avr alleles in the populations (40; M. H. Balesdent and T. Rouxel, unpublished data). The present study further points out the common occurrence of Rlm 9 in winter-type OSR cultivars; therefore, one can suppose that the absence of AvrLm9 in European populations also is due to the selection pressure exerted by $R \operatorname{lm} 9$.

In contrast to European isolates, Australian isolates were extremely diverse in terms of Avr allele combinations, and often corresponded to the most complex races. This result is consistent with the description of Australian isolates overcoming resistance in $B$. juncea $(10,19,38), B$. nigra, and B. carinata (19). All known existing virulence and avirulence alleles were found in one Australian isolate or the other, therefore substantiating the common assumption of the high level of virulence of Australian isolates $(25,32)$. It is noteworthy that 18 of the 26 races identified in the collection were defined by Australian isolates, of which 13 were from Western Australia alone. This very high degree of diversity is consistent with the description of numerous specific interactions between Australian isolates and a range of Brassica genotypes (19-21,25,29,32). Compared with European isolates, where race structure originates from the $R$ genes used in the crop, the diversity of Australian isolates may be due to the low frequency of $R$ genes in Australian spring type cultivars (42). In this respect, the sampling performed in Australia on B. napus exhibiting none or only one $R$ gene probably is very representative of the actual population.

Compared with the two oldest European isolates which harbor only two virulence alleles, the oldest Australian isolate harbors seven virulence alleles. This latter isolate was obtained from a cruciferous weed only a few years after the time oilseed rape was first grown in Australia (44) and it is tempting to postulate that, in the Australian conditions, alternate hosts, including wild mustards or mustards used as green manure, were instrumental in exerting selection pressure on more diversified Avr loci, leading to this complex race pattern. The high Avr alleles diversity of populations of L. maculans in Australia may explain the ability of the pathogen to very rapidly overcome host resistances, such as that experienced recently with the single dominant $B$. rapa subsp. sylvestris-derived resistance (29), or the earlier report by Ballinger and Salisbury (10) of an apparent reduction in the level of field resistance of some Australian cultivars. One or more previous major shifts in populations of $L$. maculans probably also have occurred in the past in Australia; for example, between the first use of cultivars such as Wesreo, carrying the Rlm4 major gene, and the present $(42,43)$.

Finally, it was more difficult to draw general conclusions on Canadian populations due to the low number of isolates analyzed here. Important regional disparities can be expected, because the four Ontario isolates were very similar to European isolates and clustered with them in a neighbor-joining dendrogram. In contrast, all the Saskatchewan isolates can be considered as a distinct population sharing the absence of AvrLm7 and AvrLm8 along with the conservation of numerous Avr alleles, such as AvrLm1, AvrLm2, AvrLm3, and AvrLm9.

Race structure disparities between the main OSR-growing areas of the world have major implications for an efficient and wellmanaged use of major $R$ genes. For example, $R \operatorname{lm} 7$ is probably a resistance source to consider in the European conditions, whereas its usefulness seems already limited in Australia or Canada. Rlml, $R \operatorname{lm} 2, R \operatorname{lm} 3$, and $R \operatorname{lm} 9$ can still be useful to help manage the disease in some parts of Canada with, however, the need to precisely define the significance in terms of Avr alleles of the recent surge of PG3 isolates observed in some parts of Canada (14). In contrast, $R \operatorname{lm} 1, R \operatorname{lm} 2, R \operatorname{lm} 3$, and $R \operatorname{lm} 9$ are fully overcome (or in the process of being) in European conditions. Finally, the existence of all virulence alleles in the Australian collection analyzed questions the feasibility of using specific major genes expressed at the leaf level in the Australian context, particularly in the perspective of a widespread deployment of non- $B$. napus cruciferous species as crops in their own right, such as for green manure crops $(1,2,11)$ and sources of industrial oils $(16,27)$. These conclusions were drawn using a very limited and heterogeneous sampling of isolates. There is now a need to develop large-scale population surveys to confirm the preliminary data described here and to provide a more extensive overview of populations to determine continent-wide or regional disparities (45). In this respect, the fully genotyped IBCN collection will be a resource for any further race structure analyses.

\section{ACKNOWLEDGMENTS}

This work was partly supported by EU contract FAIR3CT96-1669. We thank B. Letarnec, L. Coudard, J. P. Janiault, and M. Chabirand (INRA PMDV, Versailles, France) for plant management; S. Gowers (CFR, Christchurch, New Zealand) for the New Zealand isolates; B. Koopmann (University of Göttingen, Germany), M. Jedryczka (IGR-Poznan, Poland), B. Fitt (IACR, Rothamsted, UK) and J.S. Dias (ISA, Lisbon, Portugal), for the European isolates BBA62908, Raw4, UK1, and PT1, respectively; and M. Renard (INRA Le Rheu, France) for the MX lines.

\section{LITERATURE CITED}

1. Angelini, L., Lazzeri, L., Galletti, S., Cozzani, A., Macchia, M., and Palmieri, S. 1998. Antigerminative activity of three glucosinolate-derived products generated by myrosinase hydrolysis. Seed Sci. Technol. 26:771-780.

2. Angus, J. F., Gardner, P. A., Kirkegaard, J. A., and Desmarchelier, J. M. 1994. Biofumigation: Isothiocyanates released from Brassica roots inhibit growth of take-all fungus. Plant Soil 162:107-112.

3. Anonymous. 2003. Crop variety sowing guide for Western Australia 2004. N. Littlewood, ed. Dep. Agric. West. Aust. Bull. 4592.

4. Ansan-Melayah, D., Balesdent, M.-H., Buée, M., and Rouxel, T. 1995. Genetic characterization of AvrLm1, the first avirulence gene of Leptosphaeria maculans. Phytopathology 85:1525-1529.

5. Ansan-Melayah, D., Balesdent, M.-H., Delourme, R., Pilet, M. L., Tanguy, X., Renard, M., and Rouxel, T. 1998. Genes for race-specific resistance against blackleg disease in Brassica napus L. Plant Breed. 117:373-378.

6. Attard, A., Gourgues, M., Gout, L., Schmit, J., Roux, J., Narcy, J. P., Balesdent, M. H., and Rouxel, T. 2001. Molecular characterisation and polymorphism of MinLm1, a minisatellite from the phytopathogenic ascomycete Leptosphaeria maculans. Curr. Genet. 40:54-64.

7. Badawy, H. M. A., Hoppe, H. H., and Koch, E. 1991. Differential reactions between the genus Brassica and aggressive single spore isolates of Leptosphaeria maculans. J. Phytopathol. 131:109-119.

8. Balesdent, M. H., Attard, A., Ansan-Melayah, D., Delourme, R., Renard, M., and Rouxel, T. 2001. Genetic control and host range of avirulence towards Brassica napus cvs. Quinta and Jet Neuf in Leptosphaeria maculans. Phytopathology 91:70-76.

9. Balesdent, M.-H., Attard, A., Kühn, M. L., and Rouxel, T. 2002. New avirulence genes in the phytopathogenic fungus Leptosphaeria maculans. Phytopathology 92:1122-1133.

10. Ballinger, D. J., and Salisbury, P. A. 1996. Seedling and adult plant evaluation of race variability in Leptosphaeria maculans on Brassica species in Australia. Aust. J. Exp. Agric. 36:485-488.

11. Brown, P., and Morra, M. 1995. Glucosinolate-containing plant tissues as bioherbicides. J. Agric. Food Chem. 43:3070-3074. 
12. Brun, H., Levivier, S., Somda, I., Ruer, D., Renard, M., and Chèvre, A. M. 2000. A field method for evaluating the potential durability of new resistance sources: Application to the Leptosphaeria maculans-Brassica napus pathosystem. Phytopathology 90:961-966.

13. Delourme, R., Pilet-Nayel, M. L., Archipiano, M., Horvais, R., Tanguy, X., Rouxel, T., Brun, H., Renard, M., and Balesdent, M. H. 2004. A cluster of major specific resistance genes to Leptosphaeria maculans in Brassica napus. Phytopathology 94:578-583.

14. Fernando, W. G. D., and Chen, Y. 2003. First report on the presence of Leptosphaeria maculans pathogenicity group-3, the causal agent of blackleg of canola in Manitoba. Plant Dis. 87:1268.

15. Ferreira, M. E., Rimmer, S. R., Williams, P. H., and Osborn, T. C. 1995. Mapping loci controlling Brassica napus resistance to Leptosphaeria maculans under different screening conditions. Phytopathology 85:213217.

16. Fitch Haumann, B., 1991. Work continues on new oils for industrial use. Int. News on Fats, Oil, and Related Material (INFORM) 2:678-699.

17. Gall, C., Balesdent, M.-H., Desthieux, I., Robin, P., and Rouxel, T. 1995. Polymorphism of $\mathrm{Tox}^{0}$ Leptosphaeria maculans isolates as revealed by soluble protein and isozyme electrophoresis. Mycol. Res. 99:221-229.

18. Gall, C., Balesdent, M. H., Robin, P., and Rouxel, T. 1994. Tetrad analysis of acid phosphatase, soluble protein patterns, and mating type in Leptosphaeria maculans. Phytopathology 84:1299-1305.

19. Hua, L., Barbetti, M. J., and Sivasithamparam, K. 2005. Hazard from reliance on cruciferous hosts as sources of major gene based resistance for managing blackleg (Leptosphaeria maculans) disease. Field Crops Res. 91:185-198.

20. Hua, L., Damour, L., Sivasithamparam, K., and Barbetti, M. J. 2004. Increased virulence and physiological specialization among Western Australian isolates of Leptosphaeria maculans breaking down existing single dominant gene-based resistance in six cultivars of Brassica napus. Brassica 6:9-16.

21. Hua, L., Sivasithamparam, K., Barbetti, M. J., and Kuo, J. 2004. Germination and invasion by ascospores and pycnidiospores of Leptosphaeria maculans on spring-type Brassica napus canola varieties with varying susceptibility to blackleg. J. Gen. Plant Pathol. 70:261-269.

22. Jedryczka, M., Rouxel, T., and Balesdent, M. H. 1999. Rep-PCR based genomic fingerprinting of isolates of Leptosphaeria maculans from Poland. Eur. J. Plant Pathol. 105:813-823.

23. Koch, E., Song, K., Osborn, T. C., and Williams, P. H. 1991. Relationship between pathogenicity and phylogeny based on restriction fragment length polymorphism in Leptosphaeria maculans. Mol. Plant-Microbe Interact. 4:341-349.

24. Kuswinanti, T., Koopmann, B., and Hoppe, H. H. 1999. Virulence pattern of aggressive isolates of Leptosphaeria maculans on an extended set of Brassica differentials. Z. Planzenkrankh. Planzenschutz 106:12-20.

25. Kutcher, H. R., van den Berg, C. G. J., and Rimmer, S. R. 1993. Variation in pathogenicity of Leptosphaeria maculans on Brassica spp. based on cotyledon and stem reactions. Can. J. Plant Pathol. 15:253-258.

26. Langella, O. 1997. Populations. www.pge.cnrs-gif.fr/bioinfo/populations/ index.php?lang=en. Published online by CNRS (Centre National de la Recherche Scientifique).

27. Lazzeri, L., Leoni, O., Conte, L. S., and Palmieri, S. 1994. Some technological characteristics and potential uses of Crambe abyssinicia products. Ind. Crop Prod. 3:103-112.

28. Li, C. X., and Cowling, A. 2003. Identification of a single dominant allele for resistance to blackleg in Brassica napus 'Surpass 400'. Plant Breed. 112:485-488.
29. Li, H., Sivasithamparam, K., and Barbetti, M. J. 2003. Breakdown of a Brassica rapa ssp. sylvestris single dominant blackleg resistance gene in B. napus rapeseed by Leptosphaeria maculans field isolates in Australia. Plant Dis. 87:752

30. Mahuku, G. S., Goodwin, P. H., Hall, R., and Hsiang, T. 1997. Variability in the highly virulent type of Leptosphaeria maculans within and between oilseed rape fields. Can. J. Bot. 75:1485-1492.

31. Mendes-Pereira, E., Balesdent, M.-H., Brun, H., and Rouxel, T. 2003. Molecular phylogeny of the Leptosphaeria maculans-L. biglobosa species complex. Mycol. Res. 107:1287-1304.

32. Mengistu, A., Rimmer, S. R., Koch, E., and Williams, P. H. 1991. Pathogenicity grouping of isolates of Leptosphaeria maculans on Brassica napus cultivars and their disease reaction profiles on rapid-cycling Brassicas. Plant Dis. 75:1279-1282.

33. Nei, M. 1973. The theory and estimation of genetic distances. Pages 4555 in: Genetic Structure of Populations. N. E. Morton, ed. University Press of Hawaii, Honolulu.

34. Page, R. D. M. 1996. TreeView: An application to display phylogenetic trees on personal computers. Comput. Appl. Biosci. 12:357-358.

35. Pongam, P., Osborn, T. C., and Williams, P. H. 1998. Genetic analysis and identification of amplified fragment length polymorphism markers linked to the alm1 avirulence gene of Leptosphaeria maculans. Phytopathology 88:1068-1072.

36. Pongam, P., Osborn, T. C., and Williams, P. H. 1999. Assessment of genetic variation among Leptosphaeria maculans isolates using pathogenicity data and AFLP analysis. Plant Dis. 83:149-154.

37. Purwantara, A., Barrins, J. M., Cozijnsen, A. J., Ades, P. K., and Howle, B. J. 2000. Genetic diversity of isolates of the Leptosphaeria maculans species complex from Australia, Europe and North America using amplified fragment length polymorphism analysis. Mycol. Res. 104:772-781.

38. Purwantara, A., Salisbury, P. A., Burton, W. A., and Howlett, B. J. 1998. Reaction of Brassica juncea (Indian mustard) lines to Australian isolates of Leptosphaeria maculans under glasshouse and field conditions. Eur. J. Plant Pathol. 104:895-902.

39. Raymond, M., and Rousset, F. 1995. GENEPOP (version 1.2): Population genetics software for exact tests and ecumenicism. J. Hered. 86:248-249.

40. Rouxel, T., Penaud, A., Pinochet, X., Brun, H., Gout, L., Delourme, R., Schmit, J., and Balesdent, M.-H. 2003. A 10-year survey of populations of Leptosphaeria maculans in France indicates a rapid adaptation towards the Rlm1 resistance gene of oilseed rape. Eur. J. Plant Pathol. 109:871-881.

41. Rouxel, T., and Séguin-Swartz, G. 1995. International Blackleg of Crucifers Network. Blackleg News 5:15-16.

42. Rouxel, T., Willner, E., Coudard, L., and Balesdent, M. H. 2003. Screening and identification of resistance to Leptosphaeria maculans (stem canker) in Brassica napus accessions. Euphytica 133:219-231.

43. Roy, N. N. 1978. Wesreo-a blackleg resistant rapeseed. J. Agric. West. Aust. 19:42.

44. Roy, N. N., and Reeves, J. 1975. Breeding better rape and linseed for Western Australia. J. Agric. West. Aust. 16:93-97.

45. Stachowiak, A., Olechnowicz, J., Jedryczka, M., Rouxel, T., Balesdent, M. H., Happstadius, I., Gladders, P., and Evans, N. 2004. Frequency of avirulence genes in field populations of L. maculans in Germany, UK and Poland. IOBC Meeting "Integrated protection in oilseed crops." Rothamsted, UK.

46. Volke, B. 1999. Leptosphaeria maculans, der Erreger der Wurzelhals- und Stengelfäule an Raps: Verbreitung verschiedener Pathogenitätsgruppen in Europa, Quantifizierung des Befalls und Schadwirkung im Freiland. Ph.D. thesis, University of Göttingen, Germany. 